\title{
Polarization effects in nitride semiconductor device structures and performance of modulation doped field effect transistors
}

\author{
Hadis Morkoç ${ }^{\mathrm{a}, *}$, Roberto Cingolani ${ }^{\mathrm{a}, 1}$, Bernard Gil ${ }^{\mathrm{b}}$ \\ ${ }^{a}$ College of Engineering and Department of Physics, Virginia Commonwealth University, P.O. Box 843072, Richmond, VA 23284- \\ 3072, USA ${ }^{2}$ \\ ${ }^{\mathrm{b}}$ University de Montpellier II, 34095 Montpellier Cedex 5, France
}

Received 17 March 1999; accepted 13 April 1999

\begin{abstract}
Wide bandgap nitride semiconductors have recently attracted a great level of attention owing to their direct bandgaps in the visible to ultraviolet regions of the spectrum as emitters and detectors. Moreover, this material system with its favorable hetero-junctions and transport properties began to produce very respectable power levels in microwave amplifiers. If and when the breakdown fields achieved experimentally approach the predicted values, this material system would also be very attractive for power switching devices. In addition to the premature breakdown, and high concentration of defects and inhomogeneities, a number of scientific challenges remain including a clear experimental investigation of polarization effects. In this paper, following a succinct review of the progress that has been made, spontaneous and piezoelectric polarization effects and their impact on sample devicelike hetero-structures will be treated. (C) 1999 Elsevier Science Ltd. All rights reserved.
\end{abstract}

\section{Introduction}

Semiconductor nitrides such as aluminum nitride $(\mathrm{AlN})$, gallium nitride $(\mathrm{GaN})$ and indium nitride $(\mathrm{InN})$ are very promising materials for their potential use in opto-electronic devices (both emitters and detectors) and high power/temperature electronic devices as have been treated in length and reviewed recently [1-5]. These materials and their ternary and quaternary

\footnotetext{
* Corresponding author. Tel.: + 1-804-827-3765; fax: +1804-828-4269.

E-mail address: hmorkoc@vcu.edu (H. Morkoç)

${ }^{1}$ Permanent address: Department of Material Science, University of Lecce, Lecce Italy.

${ }^{2}$ http://www.vcu.edu/egrweb/vmc/research/index.html
}

alloys cover an energy bandgap range of 1.9 to $6.2 \mathrm{eV}$ which spans a color range from red (potentially) to violet. Specifically, nitrides are suitable for such applications as surface acoustic wave devices [6], UV detectors [7,8], Bragg reflectors [9], waveguides, UV and visible light emitting diodes (LEDs) [10-12] and laser diodes (LDs) [13] for digital data read-write applications. The nitride semiconductor-based LEDs are very reliable and found quick acceptance in display, lighting, indicator lights, advertisement and traffic signs/signals related applications. Lasers, as coherent sources, are crucial for high-density optical read and write technologies. Because the diffraction-limited optical storage density increases almost quadratically as the probe laser wavelength is reduced, nitride-based coherent sources at wavelengths down to near UV are attracting a good deal of attention.

0038-1101/99/\$ - see front matter (C) 1999 Elsevier Science Ltd. All rights reserved.

PII: S0038-1101(99)00146-X 
When used as UV sensors in jet engines, automobiles and furnaces (boilers), the devices would allow optimal fuel efficiency and control of effluents for a cleaner environment. Moreover, UV sensors that operate in the solar blind region (260-290 nm) would have high detectivity because the ozone layer absorbs solar radiation at those wavelengths, thus virtually eliminating radiation noise. Consequently, these detectors are expected to play a pivotal role in threat recognition aimed against aircraft $[7,8,14]$. GaN/AlGaN UV $p-i-n$ detectors have demonstrated sensitivities of about 0.12 $\mathrm{A} / \mathrm{W}$ and response speed of a few nanoseconds in 250 $\mu \mathrm{m}$ dot sizes; both values represent a quantum leap over previous capability.

GaN's large bandgap, large dielectric breakdown field, fortuitously good electron transport properties and good thermal conductivity are conducive for use in high power electronic devices [15]. Sheppard et al. [16] already reported that $0.45 \mu \mathrm{m}$ gate high power modulation doped FETs (MODFETs) on SiC substrates exhibit a record power performance. The power density of $6.8 \mathrm{~W} \mathrm{~mm}^{-1}$ in a $125 \mu \mathrm{m}$-wide device and a record total power of $4 \mathrm{~W}$ (with a power density of 2 $\mathrm{W} \mathrm{mm}^{-1}$ ) at $10 \mathrm{GHz}$ were reported. Other groups have also reported on the superior performance of GaN-based MODFETs on $\mathrm{SiC}$ and sapphire substrates with respect to competing materials, particularly at Xband and higher frequencies [16-19]. Applications include amplifiers operative at high temperatures and in unfriendly environments as well as in low-cost compact amplifiers for earthbound and space applications.

Nitride semiconductors have been deposited by vapor phase epitaxy (i.e., both hydride VPE (HVPE) and organometallic VPE (OVPE)) and in a vacuum by molecular beam epitaxy (MBE). With its innate refined control of growth parameters, in situ monitoring capability and uniformity, MBE is well suited for depositing hetero-structures and gaining insight to deposition/ incorporation mechanisms. MBE's control over growth parameters is such that any structure can be grown in any sequence. The structures for IR lasers in CD players, surface emitting vertical cavity lasers and high-performance electronic devices such as pseudomorphic MODFETs have all been produced very successfully, all of them commercially, by MBE. Nitride growth, however, requires much higher temperatures than those used in producing the conventional group III-V semiconductors for which the MBE systems were designed. In addition, it has proved difficult to provide active $\mathrm{N}$ species at sufficiently high rates for nitride growth. Despite these mechanical/engineering limitations and its relatively late entry, MBE has already played a key role on a number of fronts, such as high performance GaN-based MODFETs and fast solar blind detectors that have been discussed elsewhere [14].
Being non-centro-symmetric, nitrides exhibit large piezoelectric effects when under stress along the $c$ direction. What is not as appreciated is the spontaneous polarization at hetero-interfaces caused, in an over simplistic view, by the ionicity and uniaxial nature of the wurtzite structure. Polarization causes a sizable red shift (Stark effect) [20] in transition energies in InGaN/GaN [21,22] and AlGaN/GaN [20,23-25] quantum wells. Polarization and pyroelectric effects due to hetero-interfaces in an ionic crystal, misfit and thermal strain, anisotropy and temperature gradients have important ramifications in electronic devices, particularly in modulation doped FETs. For example, electric field caused by polarization effects can increase or decrease interfacial free carrier concentrations. As in the case of quantum wells, the literature interpretation of polarization effects in devices has in many cases been lumped into piezoelectric effects [26-28].

Despite highly imperfect material, device performance in both emitters/detectors and the microwave amplifiers has been truly outstanding. If the defects causing premature breakdown were reduced/eliminated, one would wonder whether it would be wrong at all to attempt to develop power/switching devices with large hold voltages and current handling capabilities. In this paper the topical topic of polarization issues will be visited.

\section{Polarization effects}

As alluded to earlier, observations support the presence of electric field in GaN-based hetero-structures. The genesis is two fold: piezoelectric effects and the difference in spontaneous polarization between $\mathrm{AlGaN}$ and $\mathrm{GaN}$ even in the absence of strain. Spontaneous polarization was only recently fully understood, see King-Smith and Vanderbilt [29], Resta [30] and Bernardini and Fiorentini [31]. Nitrides lack inversion symmetry and exhibit piezoelectric effects when strained along[0001]. Piezoelectric coefficients are

Table 1

Piezoelectric constants and spontaneous polarization charge in nitride semiconductors (in $\mathrm{C} \mathrm{m}^{-2}, 1 \mathrm{C} \mathrm{m}^{-2}=6.25 \times 10^{14} \mathrm{e}$ $\mathrm{cm}^{-2}$ )

\begin{tabular}{llll}
\hline & AlN & GaN & InN \\
\hline$e_{33}\left(\mathrm{C} \mathrm{m}^{-2}\right)^{\mathrm{a}}$ & 1.46 & 0.73 & 0.97 \\
$e_{31}\left(\mathrm{C} \mathrm{m}^{-2}\right)^{\mathrm{a}}$ & -0.60 & -0.49 & -0.57 \\
$P_{0}\left(\mathrm{C} \mathrm{m}^{-2}\right)$ & -0.081 & -0.029 & -0.032 \\
$e_{31}-\left(C_{31} / C_{33}\right) e_{33} \mathrm{~b}$ & -0.86 & -0.68 & -0.90 \\
\hline
\end{tabular}

\footnotetext{
${ }^{\mathrm{a}} e_{31}$ and $e_{33}$ are piezoelectric constants.

${ }^{\mathrm{b}} C_{31}$ and $C_{33}$ are elastic constants.
} 


\section{[0001] Axis}

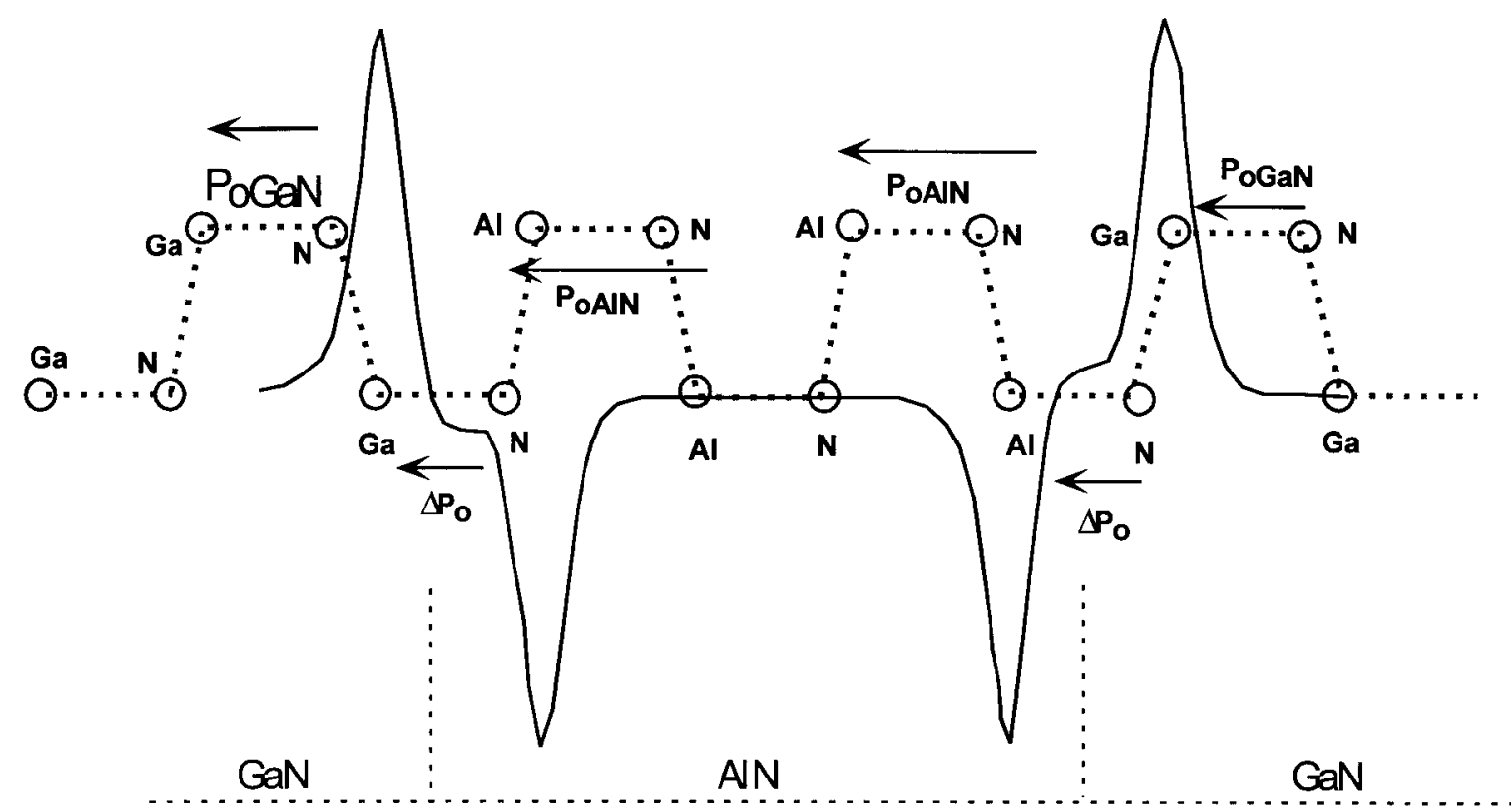

Fig. 1. Spontaneous polarization at $\mathrm{AlN} / \mathrm{GaN}$ interfaces in a heterostructure with Ga-polarity, i.e., the $c$-direction bonds point from $\mathrm{Ga}$ to $\mathrm{N}$ sites. The surface also represents the Ga layer.

almost an order of magnitude larger than in traditional III-V [32], as shown in Table 1. In addition, wurtzite GaN has a unique axis, thus allowing spontaneous polarization $\left(P_{0}\right.$ whose values are given in Table 1) even in the absence of strain. This manifests itself as polarization charge at hetero-interfaces. Caution must be exercised here as polarization effects assume insulating films. Defect laden GaN and related materials with large concentrations of free carriers hardly qualify for the simple picture that is about to be discussed. A particular concern involves the spontaneous polarization in that sharp and ideal interfaces with textbook case atomic arrangement is required for the simple description to apply. Nevertheless, it is very instructive to go through the processes involved, albeit in ideal cases.

Polarization is dependent on the polarity of the crystal, namely whether the bonds along the $c$-direction are from cation sites to anion sites or visa versa. The convention is that the [0001] axis points from the face of the $\mathrm{N}$ plane to the Ga plane and is the positive $z$ direction. In other words, when the bonds along the $c$ direction (single bonds) are from cation $(\mathrm{Ga})$ to anion $(\mathrm{N})$, the polarity is said to be Ga polarity. In this case the direction of the bonds are from $\mathrm{Ga}$ to $\mathrm{N}$ along the $c$-direction and the direction is said to be [0001] which is the $+z$ direction as shown in Fig. 1. By the same argument, when the bonds along the $c$-direction (single bonds) are from anion $(\mathrm{N})$ to cation $(\mathrm{Ga})$, the polarity is said to be $\mathrm{N}$ polarity. In this case the direction of the bonds are from $\mathrm{Ga}$ to $\mathrm{N}$ along the $c$-direction and this particular direction is said to be [000ī] direction which is the $-z$ direction. To shed further light, the Ga polarity means that if one were to cut the perfect solid along the $c$-plane where one breaks only one bond, one would end up with a Ga terminated surface.

Let us compare the relative importance of spontaneous polarization to piezoelectric polarization.

For a biaxially strained layer the effective piezoelectric polarization is given by

$P_{z}^{\text {piezo }}=\left[e_{31}-\left(C_{31} / C_{33}\right) e_{33}\right] \varepsilon_{\perp}$,

where $\varepsilon_{\perp}=\epsilon_{x x}+\epsilon_{y y}$ is the in-plane strain and $C_{31}$ and $C_{33}$ are elastic constants. The numerical values for $e_{31}$ $\left(C_{31} / C_{33}\right) e_{33}$ are listed in Table 1 using the values for elastic constants from Kim et al. [33] and piezoelectric and spontaneous polarization data from Bernardini et al.

For coherently strained $\mathrm{Al}_{x} \mathrm{Ga}_{1-x} \mathrm{~N}$ on a relaxed GaN substrate (buffer layer), the strain $\varepsilon_{\perp}$ is expected to be proportional to $x$. It is tensile and given by $\varepsilon_{\perp}=$ $2 x\left(a_{\mathrm{GaN}}-a_{\mathrm{AlGaN}}\right) / a_{A l G a N}$. In our case the amplitude of the strain reduces to $0.0495 x$ (using $3.112 \AA$ for $\mathrm{AlN} c$ plane lattice constant and $3.189 \AA$ for $\mathrm{GaN} c$-plane 
lattice constant (here $x$ is in the range from 0 to 1 and depicts the AIN mole fraction in the alloy). The piezoelectric polarization is then $P^{\text {piezo }}=-4.26 x \times 10^{-2} \mathrm{C}$ $\mathrm{cm}^{-2}$ or $2.66 x \times 10^{13} \mathrm{e} \mathrm{cm}^{-2}$ and points in the [0001] direction. The corresponding difference in spontaneous polarization between $\mathrm{Al}_{x} \mathrm{Ga}_{1-x} \mathrm{~N}$ and $\mathrm{GaN}$ is also expected to be proportional to $x$ and is given by $\Delta P^{\text {spon }}=-0.052 x\left(\mathrm{C} \mathrm{m}^{-2}\right)$ or $3.25 x \times 10^{13} \mathrm{e} \mathrm{cm}^{-2}$. Note that the two polarization effects have the same sign for Ga polarity and tensile strain in the alloy and point in the [0001] direction.

For an $\operatorname{In}_{x} \mathrm{Ga}_{1-x} \mathrm{~N}$ layer, the situation is rather different in that the differential spontaneous polarization between $\operatorname{In}_{x} \mathrm{Ga}_{1-x} \mathrm{~N}$ and $\mathrm{GaN}$ is much smaller, $\Delta P^{\text {spon }}=-0.003 x$ which translates to $1.88 x \times 10^{12} \mathrm{e}$ $\mathrm{cm}^{-2}$. Furthermore, the $\operatorname{In}_{x} \mathrm{Ga}_{1-x} \mathrm{~N}$ layer on $\mathrm{GaN}$ would be under compressive strain. The amplitude of the in plane strain is $\varepsilon_{\perp}=-0.195 x$ if 3.533 and $3.189 \AA$ for $c$-plane lattice constants for $\mathrm{InN}$ and $\mathrm{GaN}$ are used. Here $x$ is in the range from 0 to 1 and depicts the InN mole fraction and $P^{\text {piezo }}=+0.176 x$ or $1.1 x \times 10^{14} \mathrm{e} \mathrm{cm}^{-2}$. For an InN mole fraction of 0.15 , $\mathrm{P}^{\text {piezo }}=1.65 \times 10^{13} \mathrm{e} \mathrm{cm}^{-2}$. In this particular case, the piezoelectric polarization dominates and is opposite in direction to spontaneous polarization but even larger in absolute magnitude.

In the $\mathrm{AlGaN}$ case, the sign of the PE polarization is such as to produce a potential energy for electrons sloping down from the $\mathrm{Ga}$ face towards the $\mathrm{N}$ face. Thus, for a structure in which the Ga face is facing the surface, the potential will slope down from the AlGaN surface towards the $\mathrm{AlGaN} / \mathrm{GaN}$ interface and helps to drive electrons towards the two-dimensional electron gas (2DEG) forming at this interface. For example, if there is an ohmic metal contact on the AlGaN surface, electrons will flow towards the 2DEG below that layer. Consequently, for a structure with Ga polarity, the potential will slope down from the AlGaN surface towards the AlGaN/GaN interface. This potential gradient will help to drive free electrons towards the interface forming a 2DEG. In presence of an ohmic metal contact on the AlGaN surface, electrons will flow from the contact towards the $2 \mathrm{DEG}$ below that layer.

The most favorable situation for enhancing sheet carrier concentration would occur for an InGaN (under compressive strain) quantum well on top of a relaxed $n-\mathrm{GaN}$ and below an $\mathrm{AlGaN}$ barrier (under tensile strain) with the entire structure having cation (Ga) polarity. In that case, the field will slope down towards the InGaN/AlGaN interface in the quantum well and will help localize the electrons in the 2DEG. Note that the piezoelectric polarizations estimated here are based on the theoretical values for perfectly insulating materials. The polarization induced field will be screened by the carriers present in each layer. For example, if carriers flow from a metal contact towards the 2DEG, then this will set up a counteracting field. The equilibrium self-consistent field is ultimately determined by the requirement that the chemical potential for electrons (i.e. the Fermi level) must be constant throughout the structure and thus depends on the doping and band bending in the substrate and possibly in each of the layers. At the very least, one may expect these fields will be reduced by a factor corresponding to the macroscopic dielectric constant, i.e. a factor of order 10 but possibly larger if the layers acquire conductivity by free carriers. Consequently, a more realistic expectation for the effects on sheet carrier concentration is of the order of $10^{11}-10^{12} \mathrm{e} \mathrm{cm}^{-2}$.

The difference between these and traditional device structures without polarization effects is that for uniform doping concentrations, one obtains parabolically varying potentials with distance. Here, the linear terms from the polarization must be added on top of the parabolic terms. These linear terms lead to variations of the potential over a shorter distance scale determined by the thickness of the layers, while the parabolic terms correspond to the space-charge regions. Thus the linear terms may help to localize carriers if the polarity of the structure is chosen properly.

Some further words of caution about the above estimates are needed. If the $\mathrm{AlGaN}$ layers are not pseudomorphic but partially relaxed (by misfit dislocations for example), then the piezoelectric effect would be reduced but the spontaneous polarization would not. If the interfaces are not atomically sharp but exhibit a certain degree of inter-diffusion then the differences in spontaneous polarization would be reduced as well if such grading occurs over a large thickness. Finally, if domains with inverted polarity exist then the overall polarization effects may be washed out. Also note that in a inverted structure with $\mathrm{N}$ polarity towards the surface, it may be possible to create a $2 \mathrm{DHG}$ (hole-gas) at the $\mathrm{AlGaN} /$ substrate $\mathrm{GaN}$ interface, but if a $n$-type GaN layer is placed on top, a 2DEG may form on top of the AlGaN layer.

There is another effect with its genesis in the polarization effect. This is the pyroelectric effect with important implications in devices. Anisotropy in wide bandgap semiconductors is expected to cause thermal stimulus and thermal gradients. The latter is quite likely in devices where the junction temperatures are high against a relatively cool bulk. In such a case, the thermally induced electric field, pyroelectric effect, would be present with effects similar to those described above. This phenomenon has not been studied in detail in nitride semiconductors. Contrary to this lack of attention, the electric field generated by thermal gradients occurs readily in power devices and is comparable to the fields caused by applied voltages in nitride based FET like devices.

The immediate impact of this polarization is that the 


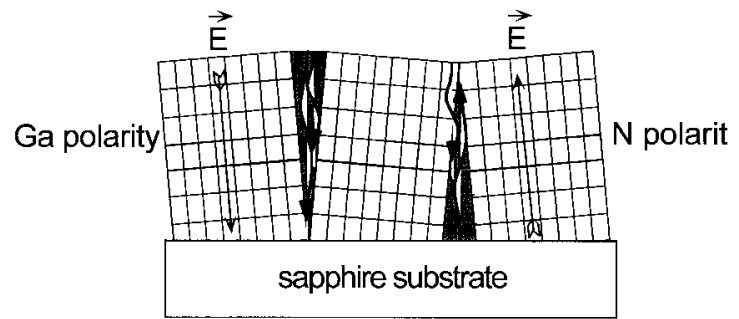

Fig. 2. Domains in $\mathrm{GaN}$, with $\mathrm{N}$ polarity (nitrogen surface layer) on the right and Ga polarity (with Ga on surface) on the left side under compressive residual strain. The arrows show the direction of the piezoelectric field in each of the domains.

field generated by this process must be considered together with that induced by the applied voltage and charge redistribution. Moreover, as alluded to earlier, free carriers can also be drawn from any shallow and weakly bound impurities and metal in contact with the semiconductor. In any case, the free carriers would tend to screen the piezoelectric-induced polarization field. An additional complicating factor in nitrides in relation to polarization is that the semiconductor tends to twist and tilt in a columnar fashion, in an effort to minimize strain as shown in Fig. 2. These columns do not necessarily have the same cation/anion ordering polarity as shown in Fig. 2. In the presence of strain, Ga polarity domains and $\mathrm{N}$ polarity domains would have opposite polarization, causing increased scattering.

Fig. 3 is a schematic representation of an ideal inversion domain boundary formed in growth along the [0001] direction. On the left of the boundary, the growth initiates with $\mathrm{N}$ and on the right it begins with

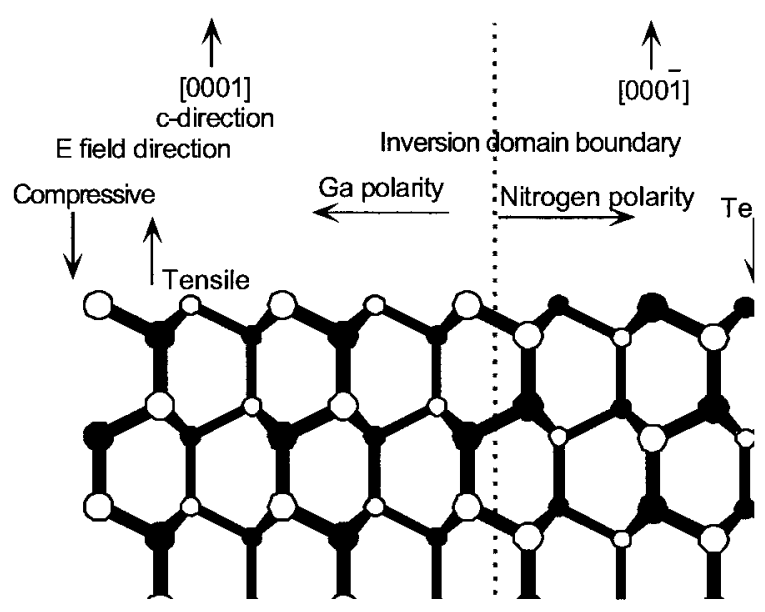

Fig. 3. Schematic representation of an ideal inversion domain boundary formed along the $c$-axis.
Ga. On the left side, the bond along the [0001] direction is from $\mathrm{Ga}$ to $\mathrm{N}$; this is called Ga polarity. On the right side, the [0001] bonds are from $\mathrm{N}$ to $\mathrm{Ga}$; this is called $\mathrm{N}$ polarity. In $\mathrm{N}$ polarity and under tensile strain, the PE field generated points toward the surface, whereas that for the Ga polarity region points in from the surface. When the strain is compressive, the direction of the field changes. Yet an additional complicating factor is the asymmetry in the barrier discontinuities between $\mathrm{GaN}$ and its binary and ternaries caused by spontaneous polarization [30]. The spontaneous polarization arises simply because of the ionicity of the bonds and the low symmetry in wurtzite structures. In fact, Bernardini et al. [31] showed that the field that occurs in quantum wells is almost completely determined by both the differential spontaneous polarization between the two semiconductors and their $\mathrm{PE}$ contribution. The field (i.e. the slope of the potential) is quite independent of the offset (i.e. the dipole discontinuity that occurs at the interface between the two materials). In other words, the dipole at the interface appears independent from the charge (monopole) accumulated at the interface.

\subsection{Polarization effect in modulation doped-Like field effect transistor structures}

Polarization effects and devices are inextricable. In devices with large concentration of free carriers, the polarization charge would be screened. One such device is the modulation doped field effect transistor (MODFET) [33], the GaAs version of which dominates the high frequency, low noise and high power (at high frequencies) device market. Recently, this device structure has been extended to include the GaN varieties. A schematic band structure representation of a $\mathrm{GaN} / \mathrm{AlGaN}$ heterostrucure used for MODFETs is shown in Fig. 4. In considering a normal MODFET (N-MODFET) structure where the larger bandgap $\mathrm{AlGaN}$ donor layer is deposited on top of a $\mathrm{GaN}$ channel layer, see Fig.5, both the spontaneous polarization and piezoelectric polarization must be accounted for. For an N-MODFET structure with Ga polarity, the potential will slope down from the surface of the $\mathrm{AlGaN}$ layer towards the $\mathrm{AlGaN} / \mathrm{GaN}$ interface and will help to drive free electrons towards the interface forming a $2 \mathrm{DEG}$ as shown in Fig. 5. For example, if there is an ohmic metal contact on the AlGaN surface, electrons will flow towards the 2DEG below the AlGaN layer from contacts. Since nitride semiconductors in question have large bandgaps, thermal generation rates are minuscule and the role played by thermally generated carriers can be ignored even at growth temperatures.

The most favorable situation for enhancing sheet carrier concentration would occur for an InGaN 


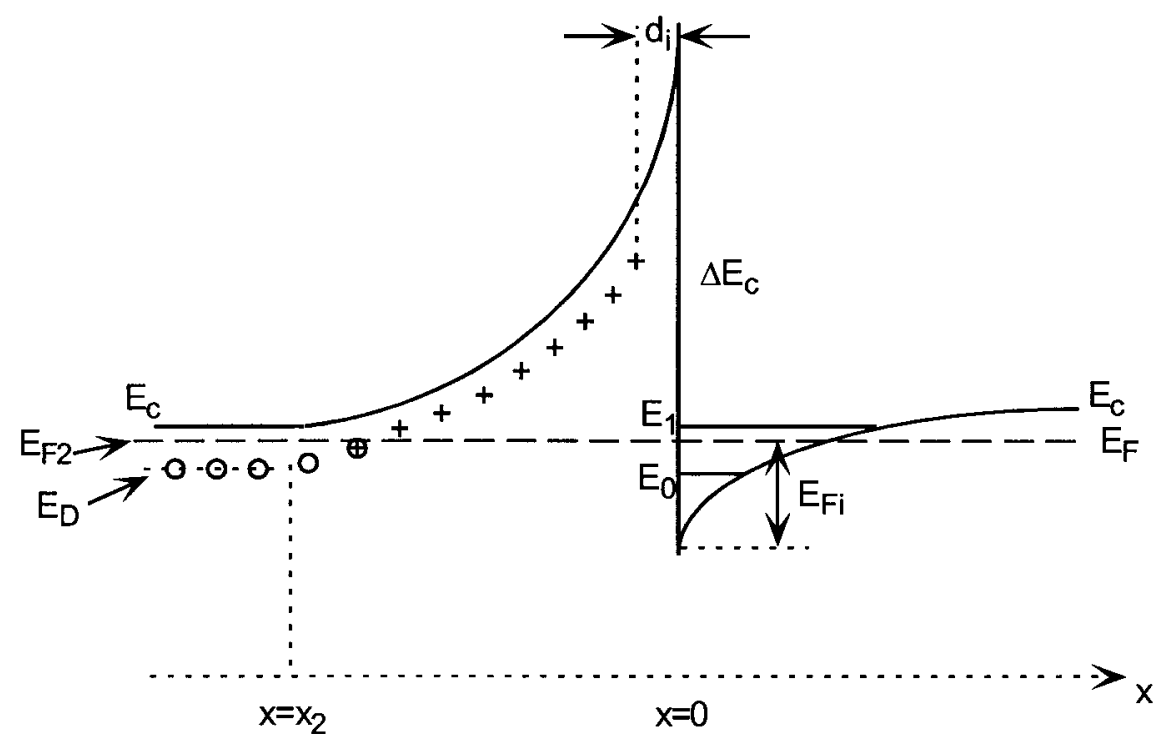

Fig. 4. Conduction band structure of a modulation-doped structure.

$\stackrel{[0001]}{\text { Ga-Polarity }}$

$\mid$ PsponAlGaNl>|PsponGaN|

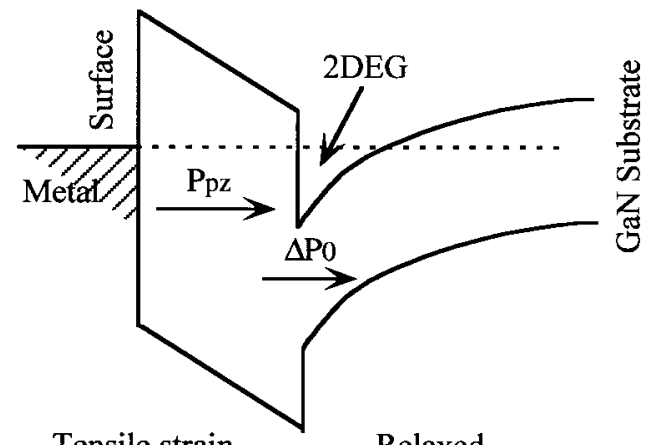

Tensile strain

Relaxed

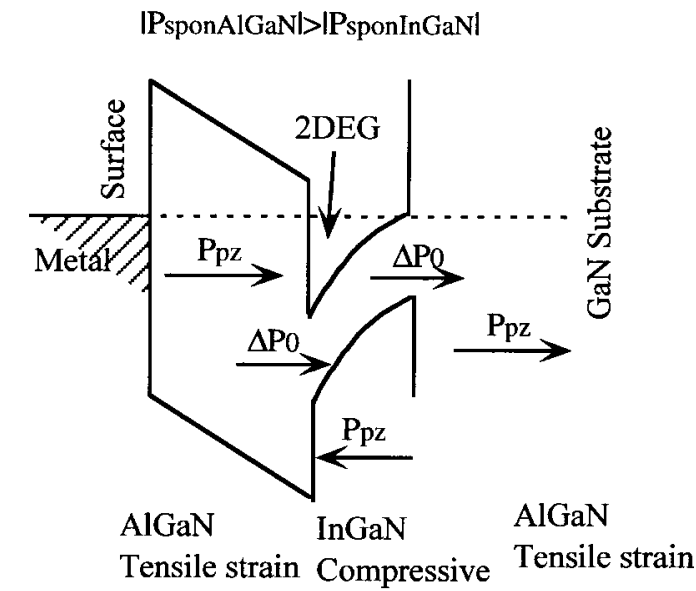

\section{Fermi level}

Fig. 5. GaN based normal modulation doped structures with Ga polarity and GaN and InGaN active layers. If the sign of strain were to change, to compressive from tensile, then the direction of the piezoelectric polarization would change. In that case the spontaneous and piezoelectric polarization charges would oppose one another and the larger one would determine whether hole or electron accumulation is favored at the interface. 


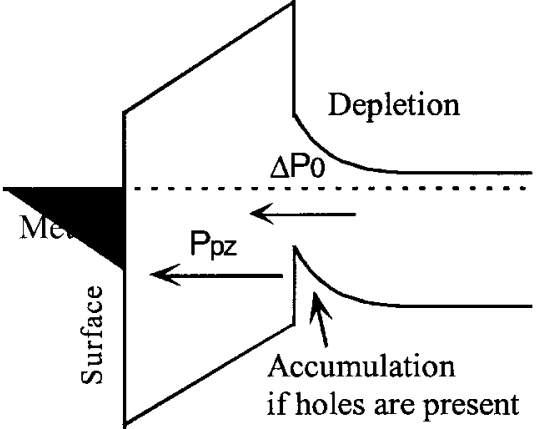

Tensile strain Relaxed

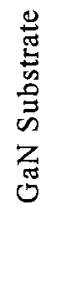

n-type GaN

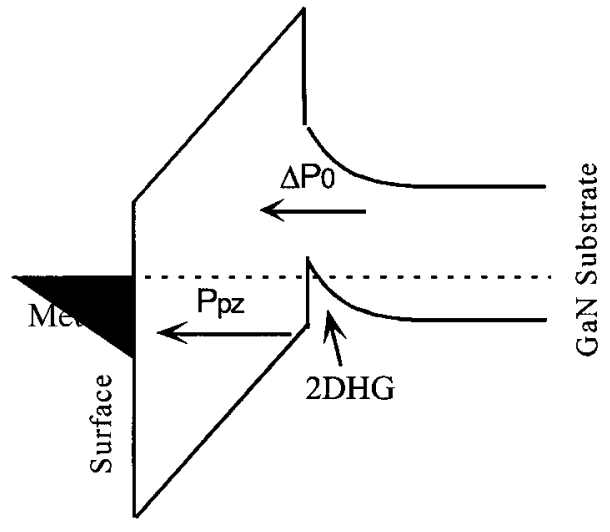

Tensile strain Relaxed

p-type GaN

-.... Fermi level

Fig. 6. AlGaN/GaN based normal modulation doped structures with $\mathrm{N}$ polarity with two cases, one for $n$-type and the other for $p$ type buffer layer. If the sign of strain were to change, to compressive from tensile, then the direction of the piezoelectric polarization would change. In that case the spontaneous and piezoelectric polarizations would oppose one another and the larger one would determine whether hole or electron accumulation is favored at the interface. Holes must be present in the system before the equilibrium shown in the figure is attained.

(under compressive strain) quantum well on top of a relaxed $n$-GaN and below an $\mathrm{AlGaN}$ barrier (under tensile strain) with the entire structure having cation (Ga) polarity as shown on the right side in Fig. 5. In that case, the field will slope down towards the InGaN/AlGaN interface in the quantum well and will help localize the carriers in the 2DEG. Note that the piezoelectric polarization vectors estimated here are based on the theoretical values for perfectly insulating materials. Since the structures available are not perfectly insulating, the field is screened by the carriers present in each layer. For example, if carriers flow from a metal contact towards the 2DEG, then this will set up a counteracting field. The equilibrium self-consistent field is ultimately determined by the requirement that the chemical potential for electrons (i.e. the Fermi level) must be constant throughout the structure and thus depends on the doping and band bending in the substrate and possibly in each of the layers. At the very least, one may expect these fields will be reduced by a factor corresponding to the macroscopic dielectric constant, i.e. a factor of order 10 , even possibly larger if the layers acquire conductivity by free carriers.

\footnotetext{
${ }^{*}$ Holes could come from contacts as in Si based p channel MOSFET also providing that truely p-type ohmic contacts are available. Metals do have large enough works functions to form barrier-less ohmic contacts on GaN.
}

Consequently, a more realistic expectation for the effects on sheet carrier concentration is of the order of $10^{11}-10^{12} \mathrm{e} \mathrm{cm}^{-2}$. A fully consistent solution of the Poisson's equation must be found with the spontaneous polarization component as interface charge in order to get the accurate charge distribution.

A case of importance in $\mathrm{GaN}$ based semiconductors is the polarity of the epitaxial layer, i.e., whether the $\mathrm{Ga}$ or the $\mathrm{N}$ plane forms the surface. It is therefore essential that the $\mathrm{N}$-face case also be considered. The case of an AlGaN (tensile strained)/GaN (relaxed) heterostructure with nitrogen polarity for an $n$-type $\mathrm{GaN}$ and for a $p$-type $\mathrm{GaN}$ buffer layer is shown for an in Fig. 6. As in the cases depicted in Fig. 5, the piezoelectric polarization and spontaneous polarization charges support one another. Unlike the Ga-polarity case, the polarization charge is such that screening charge will be made of holes if they are present in the film. If holes constitute the minority charge in the film*, then the thermal process is the means by which they would be created. However, this process in a wide bandgap semiconductor such as GaN is very slow and equilibrium condition may not be attained. If the strain in AlGaN were compressive, the direction of the piezoelectric polarization vector would change, causing the piezoelectric polarization to counter the spontaneous polarization. This would actually represent the case when the epitaxial films are relaxed at the growth temperature and upon cooling to room temperature, the 

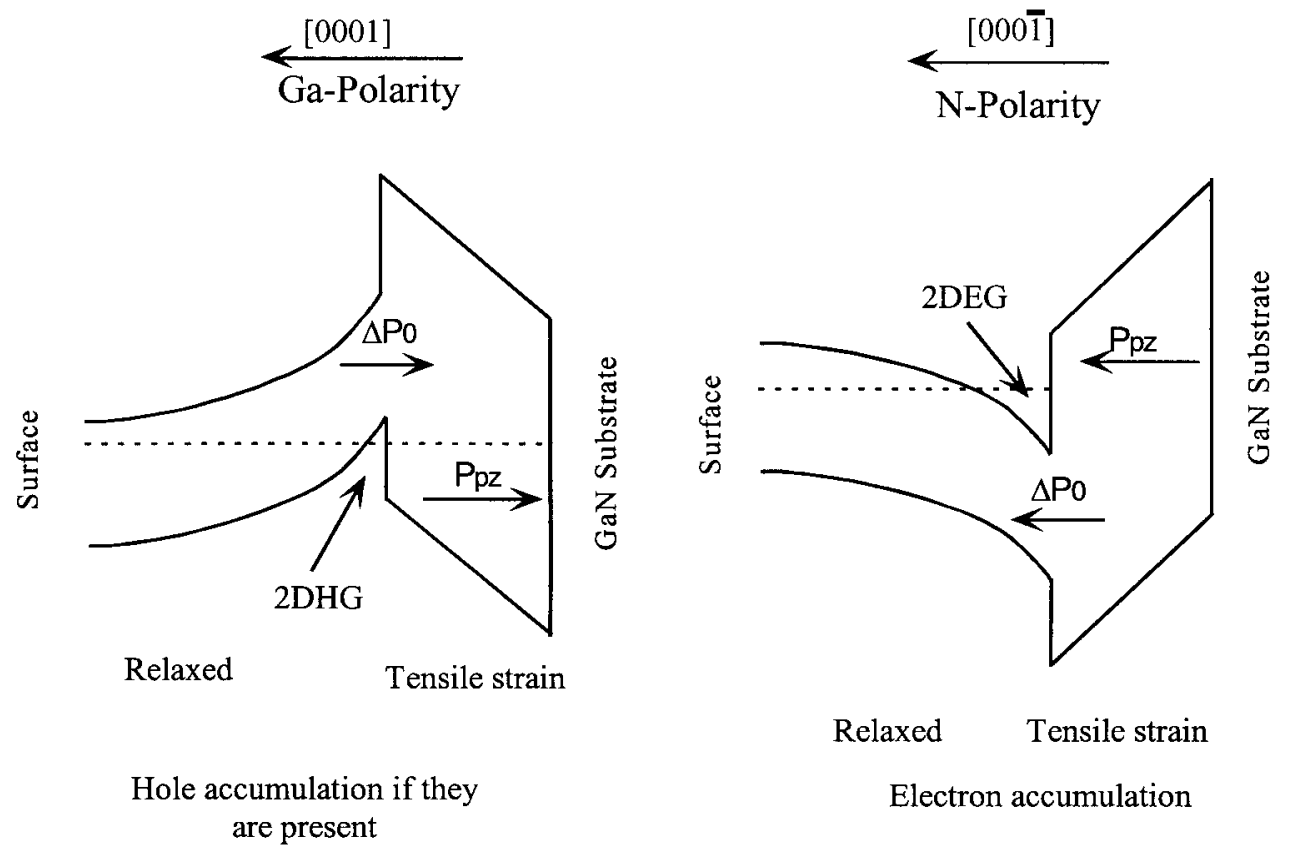

Fig. 7. AlGaN/GaN based inverted modulation doped structures with Ga and N-polarity. As can be seen, in the case of Ga polarity and tensile strain in $\mathrm{AlGaN}$, both the piezoelectric and spontaneous polarization vectors support each other leading to hole accumulation at the interface if holes are present in the system. The other source, thermal generation rate is very small and the semiconductor structure cannot be expected to reach equilibrium by this means at room temperature in a reasonable period of time. On the other hand, with N-polarity and tensile strain in $\mathrm{AlGaN}$, the structure favors electron accumulation at the interface. If the sign of strain were to change, to compressive from tensile, then the direction of the piezoelectric polarization would change. In that case the spontaneous polarization and piezoelectric polarization would oppose one another and the larger one would determine whether hole or electron accumulation is favored at the interface.

film would be under compressive strain if on sapphire substrates. This is due to the expansion coefficient of sapphire being larger than that of GaN. In such a case, the larger of the two would dominate and determines whether hole or electron accumulation would be favored. If on the other hand the film is grown on $\mathrm{SiC}$ substrates, the strain due to thermal expansion would be tensile. This would lead to the case where the piezoelectric polarization and spontaneous polarization would support one another.

Inverted modulation doped structures can also be used to interrogate the picture in effect and perhaps take advantage of the unique features present. In such a case, the AlGaN layer precedes the GaN top layer where the charge accumulation would occur. The interface between the AlGaN layer and the bottom GaN layer which is referred to as the buffer layer would be graded to prevent a normal interface from forming.

Fig. 7 shows an AlGaN/GaN based inverted modulation doped structure with $\mathrm{Ga}$ and N-polarities. As can be seen, in the case of Ga-polarity and tensile strain in $\mathrm{AlGaN}$, both the piezoelectric and spontaneous polarization vectors support each other leading to hole accumulation at the interface if holes are present in the system. Since the thermal generation rate is very small, even at high temperatures the semiconductor structure cannot be expected to reach equilibrium by this means at room temperature in a reasonable period of time. On the other hand, with $\mathrm{N}$ polarity and tensile strain in $\mathrm{AlGaN}$, the structure favors electron accumulation at the interface. If the sign of strain were to change, to compressive from tensile, then the direction of the piezoelectric polarization would change. In that case the spontaneous and piezoelectric polarization charges would oppose one another and the larger one would determine whether hole or electron accumulation is favored at the interface.

The physics literature appears to be fairly clear in that the polarization charge is a bound charge and that any free carriers act only to screen it. However, the device reports on GaN FETs have gone so far as to suggest that piezoelectric effect in and of itself is sufficient to provide the free carriers needed for devices. Moreover, polarization effects, particularly spontaneous polarization, have immense impact on measured band discontinuities. For example, the dependence of measured band discontinuities on the order in which the larger and smaller band gap semi- 


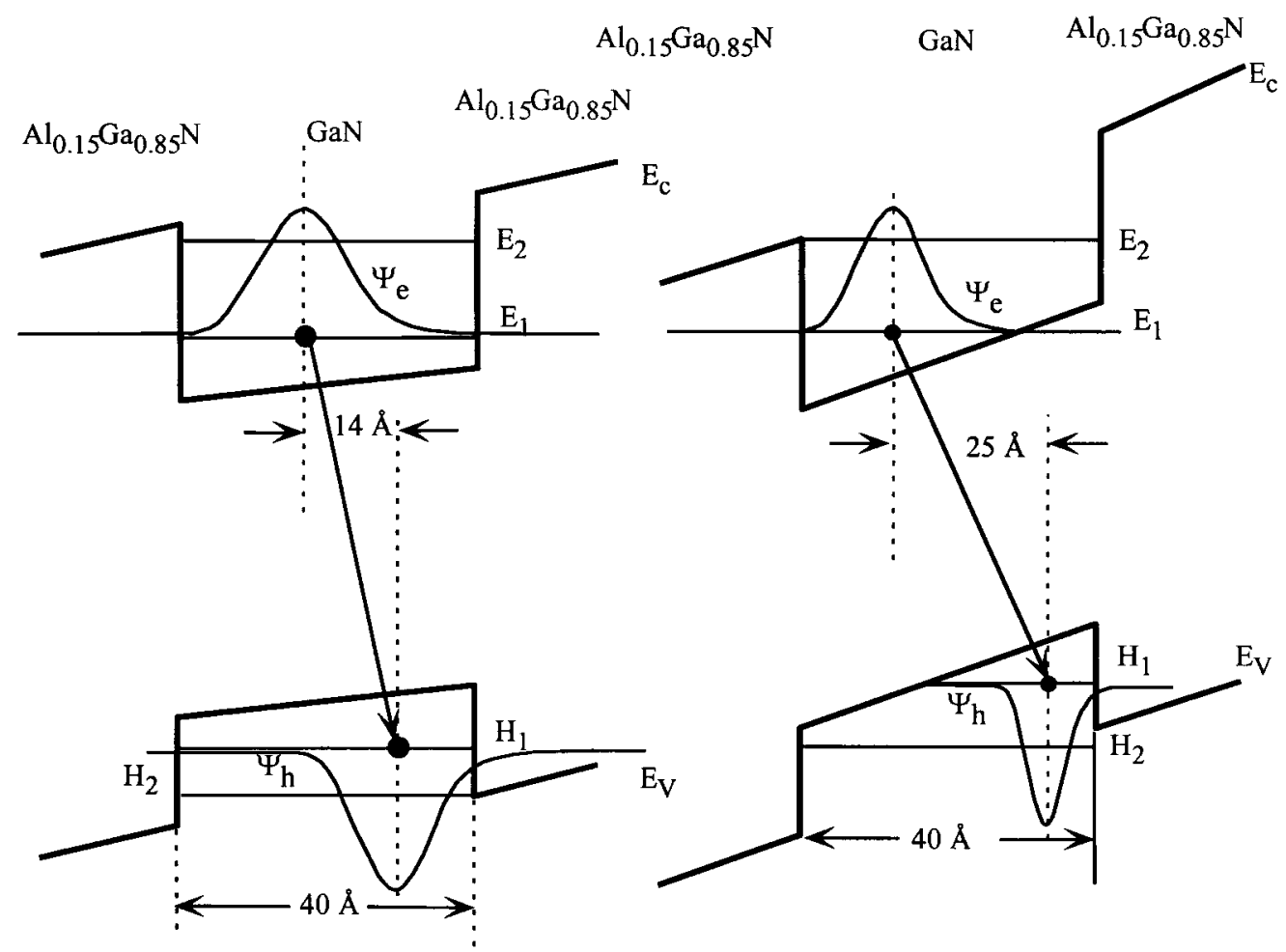

(a)

(b)

Fig. 8. The effect of the field where (a) depicts the case, e.g., shortly after the excitation pulse with large numbers of excess carriers. Case (b) depicts the evolution of the band structure some time later as the excess carriers are allowed to recombine.

conductors are grown, is one that can be attributed to polarization effects [34-36]. The complicating factor in nitrides is the lack of polarity control, $\mathrm{Ga}$ or $\mathrm{N}$ polarity and perceived lack of uniform polarity meaning presence of inversion domains.

To reiterate, as a result of polarization, the static potential at the GaN/AlN interface is different from that at the $\mathrm{AlN} / \mathrm{GaN}$ interface that gives rise to interface charge larger than the charge densities used in devices. A substantial level of effort has been expended toward determining band discontinuities, but the field is in desperate need of more in depth investigations in improved structures. The observed asymmetry in AlN/ GaN and GaN/AlN interfaces caused by spontaneous polarization is within the experimental errors of Martin et al. [35-37]. Inversion domains, see Fig. 3, combined with any strain in semiconductor nitrides lead to flipping PE fields, see Fig. 2, with adverse effects on our ability to characterize the films, let alone exploit this phenomenon for devices. Such flipping field would also cause increased scattering of carrier as they traverse in the $c$-plane. Simply put, identical device structures with different polarity layers would have widely differing performance underscoring the im- portance that these issues will have to be investigated and reconciled. The polarity mixing causes the PE induced electric field to flip from one domain to the next, causing a variation in the sheet carrier concentration along the channel of an FET like device. The same polarity mixing would have deleterious effects in the base of an HBT as well and depending on the polarity the induced field would either aid or impede minority carrier transit.

\subsection{Polarization effect in quantum wells}

Polarization effects manifest themselves in quantum wells as Stark effect as shown in Fig. 8. Time-resolved photoluminescence [23] measurements performed in GaN/AlGaN MQWs indicated that temporal evolution of the A exciton peak undergoes a red shift as the recombination depletes the excess carriers as shown in Fig. 9. If one assumes that the red shift is due to polarization induced field, one can determine the intensity of the field. To a first extent the linear approximation, that is a constant electric field, can be used. For thicker quantum wells, the quadratic approach would be more accurate. The picture may be more 


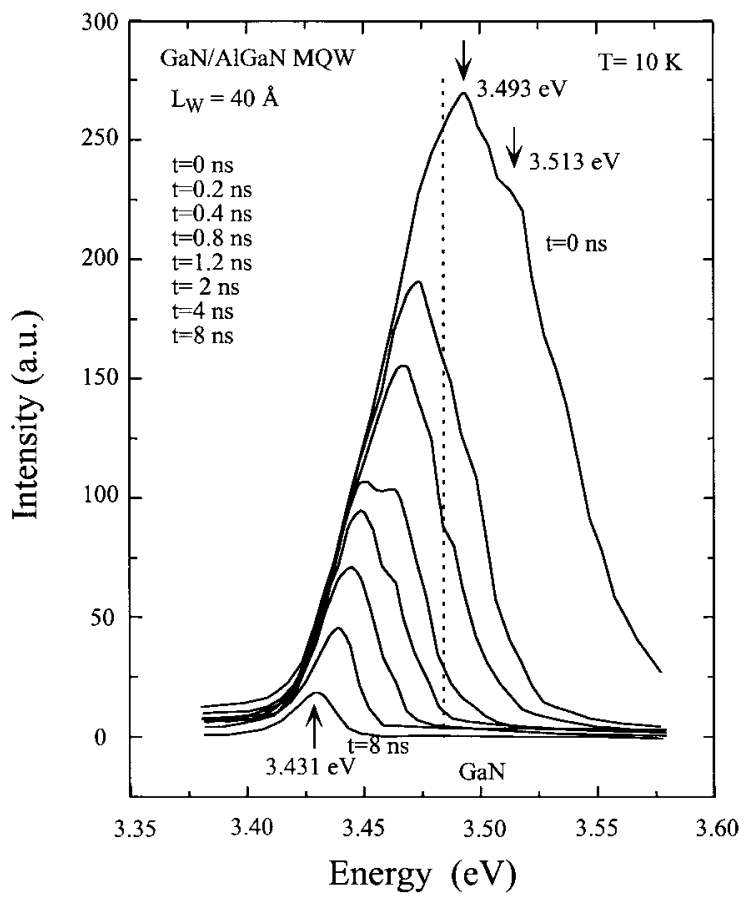

Fig. 9. Time-resolved PL signal from a $40 \AA$ well $\mathrm{AlGaN} /$ GaN MQW structure grown by MBE.

complex and suffer from variations in samples as blue shift too has been observed which may have to do with inter-well excitons and oblique excitons [37]. Though controversial, in lasers the injected carrier concentrations, even at transparency, are comparable to polarization induced charge, $10^{13} \mathrm{~cm}^{2}$, which means that the PE induced field is screened. However, the PE effect reduces the gain at lower injection levels due to, among others, reduced oscillator strength.

Using optical transitions in $\mathrm{GaN} / \mathrm{AlGaN}$, the authors and their colleagues [25] have revisited the physical origin of the built-in field and its effect on quantum wells. The results suggest that the built-in field, at least in the samples investigated, originates primarily from the spontaneous polarization charge formed at the GaN/AlGaN interfaces, with a smaller contribution from the piezoelectric field induced by strain (lattice mismatch and thermal strain or both). Such a built-in electric field causes:

1. A band bending and a red-shift of the gap which largely overcome the blue-shift expected from the quantum size effect.

2. A progressive separation of the electron and hole wave-functions with increasing the well width, resulting in the decrease of the emission strength and in the increase of the decay times.

The experiments reveal that the fundamental tran-

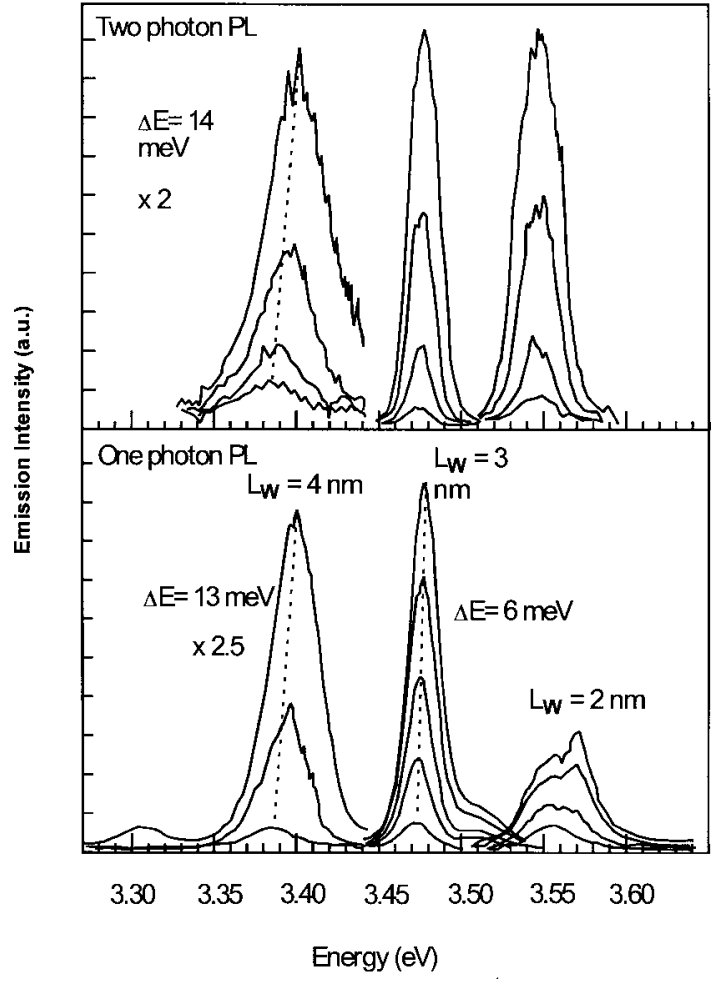

Fig. 10. One and two photon luminescence for 2, 3 and $4 \mathrm{~nm}$ $\mathrm{GaN} / \mathrm{AlGaN}$ quantum wells with injection level as the parameter.

sition of the quantum wells occurs at an energy that is well below the bulk GaN gap for well widths larger than $3 \mathrm{~nm}$ (for the specific case of $\mathrm{GaN}$ / $\mathrm{Al}_{0.15} \mathrm{Ga}_{0.85} \mathrm{~N}$ ). A total built-in field of the order of a few $\mathrm{MV} \mathrm{cm} \mathrm{cm}^{-1}$ is found and used to explain qualitatively the optical data. Moreover, a quantitative description of the red-shift and oscillator strength are obtained by means of a self-consistent tight-binding (TB) model which specifically accounts for the total built-in field and for the screening, either dielectric or induced by the photo-generated carriers. The impact of the built-in field on the actual electrostatic stability of the exciton quasi-particle in $\mathrm{GaN}$ quantum wells is also addressed.

One and two-photon absorption photoluminescence spectroscopy methods have been applied to investigate $\mathrm{GaN} / \mathrm{Al}_{0.15} \mathrm{Ga}_{0.85} \mathrm{~N}$ quantum wells of increasing thickness. The samples were grown by reactive molecular beam epitaxy on sapphire substrates. Following a chemical and in situ cleaning of $c$-plane sapphire substrates, a thin AlN buffer layer was grown at $850^{\circ} \mathrm{C}$ with ammonia as the active nitrogen source. This was followed by the growth of a $1 \mu \mathrm{m}$ thick GaN buffer layer grown at $800^{\circ} \mathrm{C}$. Finally, the quantum well region was grown. Each sample consisted of 10 GaN quan- 
tum wells. Five samples of well widths $L_{\mathrm{w}}=2,3,4,5$ and $9 \mathrm{~nm}$, were grown and analyzed. The barrier width and composition were kept constant in all samples $\left(\mathrm{Al}_{0.15} \mathrm{Ga}_{0.85} \mathrm{~N}\right.$ barriers of thickness $L_{\mathrm{b}}=10$ $\mathrm{nm})$.

A second set of samples where thick $\mathrm{Al}_{0.15} \mathrm{Ga}_{0.85} \mathrm{~N}$ buffer layers were used in an effort to cause the GaN quantum wells to be under strain were also prepared by reactive MBE. If one assumes that the GaN quantum well layers do not undergo a relaxation process, one would conclude that they would be under compressive misfit strain since the $\mathrm{GaN}$ lattice constant is smaller than that of $\mathrm{Al}_{0.15} \mathrm{Ga}_{0.85} \mathrm{~N}$. From the thermal expansion coefficient point of view, the situation is different in that all the layers, wells, barriers and the buffer layer, would be under compressive strain. Unlike the case where GaN buffer layers were used, in the case of $\mathrm{Al}_{0.15} \mathrm{Ga}_{0.85} \mathrm{~N}$ buffer layers, the piezoelectric field will be induced by misfit strain in the wells themselves. The thermal mismatch strain induced piezoelectric field will be across the entire layer structure due to the residual strain.

Linear luminescence was excited either by the 325 $\mathrm{nm}$ line of a cw He-Cd laser (10 $\mathrm{mW}$ power) or by the $337.1 \mathrm{~nm}$ line of a pulsed $\mathrm{N}_{2}$ laser $(300 \mathrm{~kW}$ peak power), in order to study the density-dependent screening of the built-in field generated by the photo-generated carriers. Two-photon absorption induced luminescence was also measured in order to make sure that the observed blue-shift was not due to band-filling of hot electron-hole pairs and to avoid the undesired occurrence of localized-states and impurity related emission in the spectra. In these experiments, the halfgap excitation provided by the tunable output of a pulsed parametric oscillator was tuned at the fundamental $n=1$ electron-heavy-hole resonance $\left(2 \hbar \omega=E_{1 \mathrm{e} 1 \mathrm{~h}}\right)$ of the quantum well. Time-resolved luminescence was also measured by using the third harmonic of a ps Ti:sapphire laser and a streak-camera. All measurements were performed at $10 \mathrm{~K}$.

In Fig. 10 we display a few representative PL spectra recorded at $10 \mathrm{~K}$ under one- and two-photon absorption excitation and for different power densities in samples with GaN buffer layers. First of all, we note that the quantum well emission is dominant and no barrier luminescence or impurity related emission is observed under pulsed excitation. The emission energy decreases with increasing well width, as expected from the quantum size effect. However, we note that for large well widths the $E_{1 \mathrm{e} \text { h }}$ transition falls below the bulk $\mathrm{GaN}$ gap $(3.51 \mathrm{eV}$ at $10 \mathrm{~K})$. In order to eliminate possible extrinsic recombination processes, we have performed two-photon absorption experiments with approximately half-gap excitation $\left(2 \hbar \omega \approx E_{1 \mathrm{e} 1 \mathrm{~h}}\right)$. Both the linear and non-linear spectra exhibit the same energy positions and line-widths for the quantum well

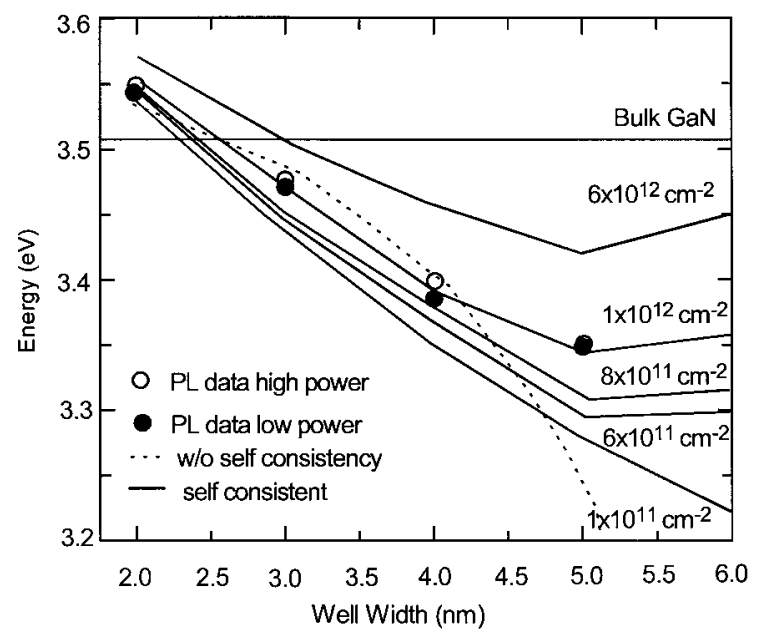

Fig. 11. The transition energies in GaN/AlGaN quantum wells as a function of well thickness. The solid and open circles indicate results with lower and higher power, respectively. Calculated values for the indicated injection levels are also shown with very good agreement with experiments.

structures under investigation, indicating that carrier heating or any possible extrinsic effects do not influence the luminescence spectra of these samples. Furthermore, we note that all samples exhibit a substantial blue-shift with increasing photo-generation rate, under both one- and two-photon absorption excitation. This suggests a screening induced blue shift caused by the partial compensation of the Stark effect by the photo-generated carriers. It is important to mention that the emission spectra do not show any significant intensity dependence under low-power cw excitation, suggesting that at low photo-generation rates the screening of the built-in field is negligible and is of no consequence.

In Fig.11, we display the progressive reduction of the emission efficiency and the increase of the decay times occurring with increasing well width in samples with GaN buffer layers. In an effort to shed light on the polarization issue, the total polarization charge can be written as $P_{\text {tot }}=P_{\text {piezo }}+P_{\text {spont }}$, where $P_{\text {piezo }}$ is the piezoelectric charge caused by the lattice mismatch $(\mathrm{lm})$ strain and by the thermal strain (ts) $\left(P_{\text {piezo }}=P_{\mathrm{lm}}+P_{\mathrm{ts}}\right]$, whereas $P_{\text {spont }}$ represents the spontaneous polarizability of the GaN/AlGaN interface, as clearly demonstrated by the recent works of Bernardini et al. [30,31]. In Fig. 12, we show the data similar to those shown in Fig. 11 with the inclusion of samples on $\mathrm{Al}_{0.15} \mathrm{Ga}_{0.85} \mathrm{~N}$ buffer layers as well. Again the calculated transition energies with increasing injection levels are shown.

The afore-mentioned experimental results support that the built-in field originates primarily from the 


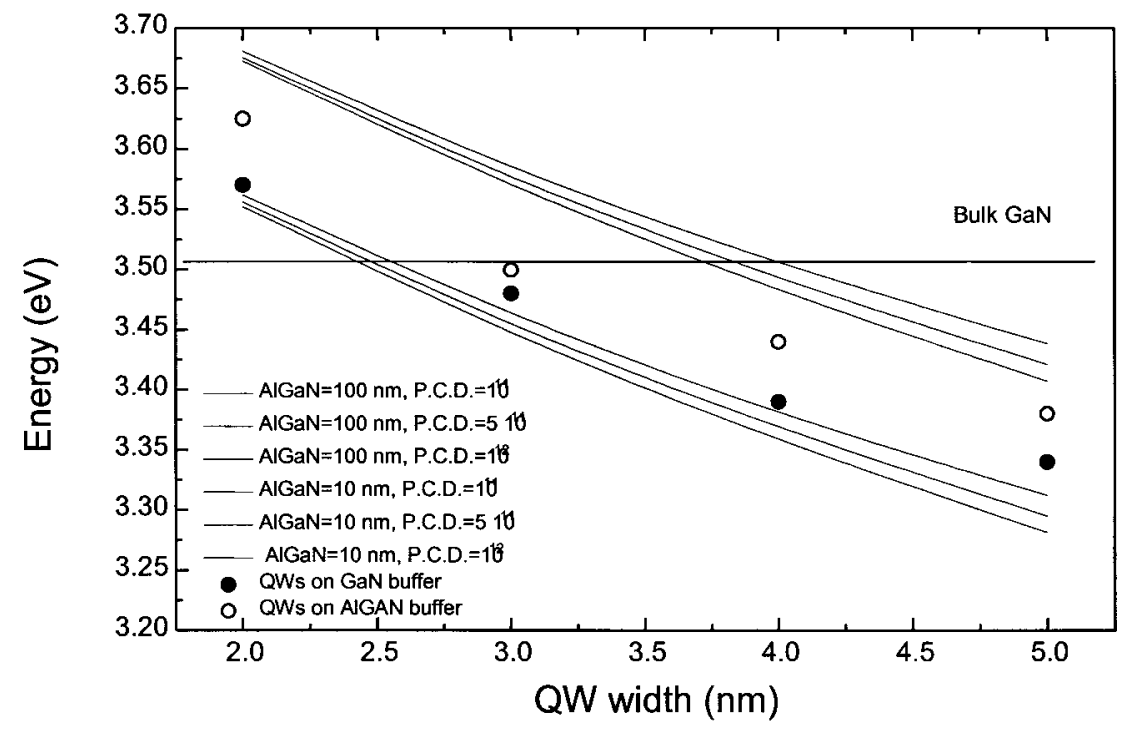

Fig. 12. The transition energies in GaN/AlGaN quantum wells prepared on $\mathrm{GaN}$ and $\mathrm{AlGaN}$ buffer layers as a function of well thickness. The latter has the same composition as the barrier material, $\mathrm{Al}_{0.15} \mathrm{Ga}_{0.85} \mathrm{~N}$. The solid and open circles indicate results with $\mathrm{GaN}$ and $\mathrm{Al}_{0.15} \mathrm{Ga}_{0.85} \mathrm{~N}$ buffer layers, respectively. Calculated values for the indicated injection levels are also shown with very good agreement with experiments.

spontaneous polarization charge formed at the $\mathrm{GaN} /$ AlGaN interfaces with a minor contribution from the piezoelectric field induced by strain (either lattice mismatch and thermal strain) as has been predicted. The experimental data, shown in Figs. 10-12, reveal that the fundamental transition of the quantum wells occurs at an energy that is well below the bulk GaN gap for well widths larger than $3 \mathrm{~nm}$ (for the specific case of $\mathrm{GaN} / \mathrm{Al}_{0.15} \mathrm{Ga}_{0.85} \mathrm{~N}$ ). Care was taken to assure that the transitions observed are of intrinsic origin by performing two-photon absorption experiments. Calculated transition energies with corresponding carrier densities depicting the blue shift caused by screening are also shown. The dotted line indicates the analytical calculations whereas the others represent self-consistent calculations.

As recently demonstrated by Bernardini et al., for an alternating sequence of wells (w) and barriers (b) the total electric field in the well can be calculated as

$$
\begin{aligned}
& F_{\mathrm{w}}=-4 \pi L_{\mathrm{b}}\left(P_{\mathrm{tot}}^{\mathrm{w}}-P_{\text {tot }}^{\mathrm{b}}\right) /\left(L_{\mathrm{w}} \varepsilon_{\mathrm{b}}+L_{\mathrm{b}} \varepsilon_{\mathrm{w}}\right), \\
& F_{\mathrm{b}}=-4 \pi L_{\mathrm{w}}\left(P_{\text {tot }}^{\mathrm{b}}-P_{\text {tot }}^{\mathrm{w}}\right) /\left(L_{\mathrm{b}} \varepsilon_{\mathrm{w}}+L_{\mathrm{w}} \varepsilon_{\mathrm{b}}\right),
\end{aligned}
$$

where $\epsilon_{\mathrm{w}, \mathrm{b}}$ is the dielectric constant of the well and barrier layer. The piezoelectric charge induced by the in-plane lattice mismatch can be found from Eq. (1) using the in-plane strain for $\mathrm{AlGaN} / \mathrm{GaN}$ as $\epsilon_{\perp}=2 x\left(a_{\mathrm{GaN}}-a_{\mathrm{AlGaN}}\right) / a_{\mathrm{AlGaN}}$ where $\mathrm{AlGaN}$ is pseudomorphically strained on a relaxed GaN substrate. Thick GaN buffer layers ensures that the GaN quan- tum wells are not under misfit strain. Moreover, since the $\mathrm{Al}_{0.15} \mathrm{Ga}_{0.85} \mathrm{~N}$ layers are thin and grow pseudomorphically, they undergo a tensile in-plane strain $\epsilon_{\perp}=0.742 \%$. This results in a piezoelectric polarization charge $P_{\mathrm{lm}}^{\mathrm{b}}=0$ in the wells and $P_{\mathrm{lm}}^{\mathrm{b}}=-3.99 \times 10^{12} \mathrm{e}$ $\mathrm{cm}^{-2}$ in the $\mathrm{Al}_{0.15} \mathrm{Ga}_{0.85} \mathrm{~N}$ barriers. The thermal strain amounts to some $0.03 \%$, resulting in an additional polarization charge of the order of $P_{\mathrm{ts}}^{\mathrm{w}}=+2.57 \times 10^{11}$ e $\mathrm{cm}^{-2}$. For spontaneous polarization, we take the recent data of Bernardini et al. leading to $P_{\mathrm{sp}}^{\mathrm{w}}=-1.81 \times 10^{12} \mathrm{e} \mathrm{cm}^{-2}$ and $P_{\mathrm{sp}}^{\mathrm{b}}=-7.64 \times 10^{12} \mathrm{e}$ $\mathrm{cm}^{-2}$, the latter value being obtained by linear interpolation of the $\mathrm{GaN}$ and $\mathrm{AlN}$ values $\left(P_{\mathrm{sp}}=-5.09 \times 10^{13} \mathrm{e} \mathrm{cm}^{-2}\right.$ in $\left.\mathrm{AlN}\right)$. The differential spontanous polarization between the $\mathrm{Al}_{0 .}{ }_{1} \mathrm{Ga}_{0.85} \mathrm{~N}$ barrier and the GaN well is then $-5.83 \times 10^{12} \mathrm{e} \mathrm{cm}^{-2}$. By using these data and Eq.(2), one can calculate the built-in field in the different samples, which turns out to vary in the range $1-3 \mathrm{MV} \mathrm{cm}^{-1}$ depending on the actual well width. Neglecting high-field effects and corrections for self-consistency, the red-shift caused by the built-in field in the wells is given by the quadratic Stark effect [38,39].

The measured ground level transition energies and those calculated in the square well approximation with inclusion of the Stark shift are in very good agreement. The agreement between theoretical and experimental data is very reasonable, especially if we take into account that the nominal growth parameters were used in the calculations, without any fitting parameter. 
The agreement between the calculated and measured data can in fact be substantially improved by the use of a self-consistent tight-binding (TB) model [40]. The tight-binding model is used to describe the electronic structure in the entire Brillouin zone, up to several eV above the fundamental gap, thus overcoming the wellknown limitations of the envelope function approach. For the specific case of nitride semiconductors, the parameters of our empirical TB model were determined by fitting the band-structure of Ref. [41]. For the self-consistent calculations the electron and hole quasi-Fermi levels are calculated for a given photoinjected charge density. The resulting electron and hole distribution functions ( $n$ and $p$, respectively) are used to solve the Poisson equation. The Poisson equation is solved assuming zero-field at the boundaries. The obtained potential is thus inserted into the TB Schroedinger equation that is solved to get the energies and wave-functions. The new quasi-Fermi levels are thus recalculated and the procedure is iterated until self-consistency is achieved. The agreement between the self-consistent calculations and the experimental data is very good both in terms of the trend and absolute values.

\section{Modulation doped field effect transistors}

Modulation doped field effect transistors which were originally developed in the GaAs/AlGaAs material system, gained a rapid foothold in high-speed device arena [33]. In particular, a variant of this technology that utilized a coherent InGaAs active layer quickly outperformed many other devices competing for the high speed, low noise and high power sector [42]. Recently, the same device concept has been extended to the GaN/AlGaN system with impressive power results [43] and eventually to the point where record power densities are obtained. Simplified analytical descriptions of MODFET operation have been developed which show quantitatively the effect of charge stored at the hetero-interface on mobility and carrier velocity. The analytical model of Drummond et al. [44] describes MODFET operation well. The model provides analytical expressions that relate the channel charge to the gate and drain potentials and predicts the current voltage characteristics. In short, the MODFET provides large mobile carrier concentrations in close proximity to the gate, which was previously unachievable. A similar model [45] has been developed for GaN/AlGaN MODFETs and used to predict their performance. In short, the favorable velocity field characteristics of $\mathrm{GaN}$ point to performance that is competitive with GaAs even in terms of small signal properties. In terms of power performance, GaAs devices are supplanted by $\mathrm{GaN}$ devices with increasing margin as the nitride technology evolves. There are, however, anomalies such as high- frequency current collapse/degradation that must first be understood and circumvented before the intrinsic properties of $\mathrm{GaN}$ can be fully exploited.

In addition to the small signal description, an accurate modeling of device power is very useful. In high power semiconductor devices, it is imperative that the effect of temperature on device performance is accounted for accurately. As in small signal modeling, the first step in power modeling is to establish the basic device geometrical factors that are needed to calculate the current voltage characteristics. Once these are known, the output characteristics superimposed with the load line can be used to estimate the power level that can be obtained from the device provided that it is not limited by the input drive. In class A operation, the maximum power that can be expected from the drain circuit of a device is given by

$P_{\max }=I_{\text {dson }}\left(V_{\mathrm{b}}-V_{\text {knee }}\right) / 8$,

where $I_{\mathrm{dson}}$ is the maximum drain current (this is the drain current with a small positive voltage on the gate electrode), $V_{\mathrm{b}}$ is the drain breakdown voltage and $V_{\text {knee }}$ is the knee voltage as shown in Fig. 4. The allowable positive gate voltage $(\approx 1 \mathrm{~V})$ will depend on the channel doping and the work function of the gate metal. The positive gate voltage is limited by the onset of forward Schottky-diode current. The DC load line would be used in a class A rf amplifier with the maximum drain voltage $V_{\mathrm{d}}=V_{\mathrm{b}} / 2$. The slope of the load line is $1 / R_{\mathrm{L}}$ where $R_{\mathrm{L}}$ is the value of the load resistance at the output of the FET. What can be gleaned from Eq. (3) is that $V_{\mathrm{b}}$ and $I_{\mathrm{d} s o n}$ must be made as large as possible. The utility of wide bandgap semiconductors such as $\mathrm{GaN}$ in this juncture is that the drain breakdown voltage is larger than that in conventional group III-V semiconductors. In general, the drain can be swung to voltages up to $80 \%$ of the drain breakdown for a $20 \%$ margin of safety. It should be pointed out that the maximum drain current in nitride semiconductor-based MODFETs is in the same ballpark as that of more conventional semiconductors. This implies that increased power handling capability is a direct result of large breakdown voltages and thermal conductivity and the fact that higher junction temperatures can be tolerated. Ability to increase drain bias increases the load resistance and makes it easier to impedance match, particularly in devices with large gate widths.

In power devices, power dissipation within the device increases the junction temperature and alters the output characteristics. On the one hand, higher junction temperatures with respect to the case temperature would enhance the heat dissipation to the power of four of the temperature differential, but along with it 
come reduced current and increased series resistances, which in turn increase the heat dissipation. Moreover, the thermal conductivity of the semiconductor decreases with increased temperature, exacerbating the situation. Consequently, the effect of junction temperature on the output characteristics must be taken into consideration. Temperature-dependent material parameters, if known, can be used to calculate the output characteristics with respect to temperature. However, a more pragmatic approach, particularly when the aforementioned parameters and or models required are not available, can be taken in which one measures the output characteristics of the device under consideration as a function of temperature. The junction temperature is critically dependent on the substrate thermal conductivity that is available for various substrates including $\mathrm{GaN}$ [1]. The functional dependence of thermal conductivity on temperature is

$\chi(T)=\chi\left(T_{0}\right)\left(T / T_{0}\right)^{-r}$,

where the coefficient $r$ is $0.559,0.443,0.524$ and 0.544 for $\mathrm{Si}, \mathrm{GaAs}, \mathrm{SiC}$ and sapphire, respectively [46]. Thermal conductivity of sapphire, SiC, GaAs and $\mathrm{Si}$ as a function of temperature are shown in Fig. 13. In Fig. 13, $\chi\left(T_{0}\right)$ has also been appropriately reduced to account for the doping of the substrate material.

\subsection{AlGaN/GaN modulation doped field effect transistors}

To reiterate, MODFET's performance is due to the conduction channel that allows large sheet carrier concentrations to be maintained and its unique capacitance-voltage relationship [47]. Moreover, spatial separation of scattering centers (such as ionized donors) from the electrons leads to low field transport void of ionized impurity scattering. As alluded to earlier at some length, what is somewhat unique to $\mathrm{GaN}$ and its alloys is their spontaneous polarization and the strain-induced piezoelectric effect $[36,48,49]$ that causes redistribution of mobile and weakly bound charge and charge collected from metal contacts while the semiconductor attempts to screen the polarization charge.

Stengel et al. [45] have recently modeled electronic properties of modulation-doped structures based on the III-nitride semiconductor system. The structure considered was a wurtzitic $\mathrm{Al}_{x} \mathrm{Ga}_{1-x} \mathrm{~N} / \mathrm{GaN}$ normal MODFET. Because of the conduction band discontinuity, the electrons diffusing from the larger bandgap $\mathrm{AlGaN}$ into the smaller bandgap $\mathrm{GaN}$ form a triangular quantum well at the $\mathrm{Al}_{x} \mathrm{Ga}_{1-x} \mathrm{~N} / \mathrm{GaN}$ interface, which is the hallmark of MODFETs. The source and drain contacts were assumed to penetrate down to the GaN layer that hosts the 2DEG.

\subsection{Experimental performance of GaN modulation doped field effect transistors}

Initial GaN MODFETs utilized the background donors in the AlGaN layer, the density of which is not controllable, to say the least and any other free and weakly bound electrons drawn to the interface. Congruent with the early stages of development and the defect-laden nature of the early GaN and AlGaN layers, the MODFETs exhibited very low transconductances and a low-resistance and a high-resistance state before and after the application of a high drain voltage $(20 \mathrm{~V})$. As in the case of GaAs/AlGaAs MODFETs, hot electron trapping in the larger bandgap material at the drain side of the gate is primarily responsible for the current collapse. The negative electron charge accumulated because of this trapping causes a significant depletion of the channel layer, more probably a pinchoff, leading to a drastic reduction of the channel conductance and the decrease of the drain current. This continues to be effective until the drain-source bias is substantially increased, leading to a space-charge injection and giving rise to an increased drain-source current.

With improvements in the materials quality available, the transconductance, current capacity and drain breakdown voltage are all increased to the point that GaN-based MODFETs are now strong contenders in the arena of high power devices/amplifiers, particularly at X-band and higher frequencies. As is the case for any FET device structure, improved and high resistivity buffer layers have once again played a pivotal role. For chronological purposes, a brief review of the latest class of MODFETs with high transconductances and current levels is given below.

Credit to the GaN material system, MODFETs with a gate length of $2 \mu \mathrm{m}$, gate width of $40 \mu \mathrm{m}$ and the drain-source separation of $4 \mu \mathrm{m}$ exhibited drain currents of approximately $500 \mathrm{~mA} \mathrm{~mm} \mathrm{~m}^{-1}$ and extrinsic transconductances of approximately $g_{\text {em }}=185 \mathrm{mS}$ $\mathrm{mm}^{-1}$ which were unprecedented. The drain breakdown voltage for $1 \mu \mathrm{m}$ gate to drain spacing was approximately $100 \mathrm{~V}$, the exact value depending on the layer design and quality of the layered structure. Although terms such as piezoelectric doping are being used rather liberally to increase the sheet carrier concentration, ultimately regardless of the source of the carriers, the strength of the electric field that can be tolerated under the gate without excessive leakage will set an upper limit on the number of carriers that can accumulate at the interface and be modulated. Use of multi-2DEG structures is one obvious method to increase the current capability of MODFETs and they 
have been employed. In those cases, the GaN layer is straddled by two doped AlGaN barriers that donate electrons to the channel, thus increasing the number of electrons available for current conduction. In double interface structures by Hall effect measurement, the mobility and sheet carrier densities in the $2 \mathrm{DEG}$ were about $304 \mathrm{~cm}^{2} / \mathrm{V}$ s and $3.7 \times 10^{13} \mathrm{~cm}^{-2}$, respectively, at room temperature. The sheet carrier concentration may have been affected by piezoelectric effect. A number of double hetero-channel MODFETs (DHCMODFETs) with gate lengths of $1.5-1.75 \mu \mathrm{m}$ and a gate width of $40 \mu \mathrm{m}$ have been reported.

The maximum drain saturation current $I_{\mathrm{DS}}$ corresponding to a drain-source voltage $V_{\mathrm{DS}}=7 \mathrm{~V}$ and gate source voltage $V_{\mathrm{GS}}=3.5 \mathrm{~V}$ in a DHCMODFET is about $1100 \mathrm{~mA} \mathrm{~mm}^{-1}$, albeit at forward biased gate voltages, which is important because in high power devices, the input is momentarily forward biased. The DHCMODFET has a room temperature extrinsic transconductance of $g_{\mathrm{m}}=270 \mathrm{mS} \mathrm{mm}{ }^{-1}$. The value of the total resistance $R_{\mathrm{T}}$ extracted from the linear region of the $I-V$ curves is $4 \Omega \mathrm{mm}^{-1}$. Near pinch-off, the drain breakdown voltage is about $80 \mathrm{~V}$, indicating excellent power potential of the device. These devices maintain reasonable output characteristics at temperatures as high as $500^{\circ} \mathrm{C}$ with maximum drain current and extrinsic transconductance values of $380 \mathrm{~mA}$ $\mathrm{mm}^{-1}$ and $70 \mathrm{mS} \mathrm{mm} \mathrm{m}^{-1}$, respectively. These measurements were made in a nitrogen-pressurized container to avoid possible oxidation of the contacts and probes. Cooling to room temperature restored the characteristics, which demonstrates the robustness of this material system and of the metallization employed. It should be noted, however, that high power operation requires large drain breakdown voltages with the added benefit of having large output resistances, which ameliorates impedance matching.

MODFETs have progressed to a point where microwave measurements have been performed on a variety of devices. Astonishingly, devices with gate length as wide as $2 \mu \mathrm{m}$ and as narrow as about $0.2 \mu \mathrm{m}$ have been explored with remarkable performance. For example, a typical MODFET structure with a $2 \mu \mathrm{m}$ gate length has been tested for small-signal $S$-parameters at bias conditions used for the power measurements (i.e., $15 \mathrm{~V},-2.5 \mathrm{~V}$ and $20 \mathrm{~mA}$ for the drain voltage, gate voltage and drain current, respectively). The unity current gain cut-off frequency $\left(f_{\mathrm{t}}\right)$ and maximum frequency of oscillation $\left(f_{\max }\right)$ were 6 and 11 $\mathrm{GHz}$, respectively, at both 15 and $30 \mathrm{~V}$ bias. Values of $f_{\mathrm{t}}$ and $f_{\max }$ in excess of 70 and $100 \mathrm{GHz}$ have been reported for short channel (about $0.2 \mu \mathrm{m}$ ) devices, respectively. The power measurements for $2 \mu \mathrm{m}$ devices were taken at $4 \mathrm{GHz}$ with the input power swept from 5 to $18 \mathrm{dBm}$ in 14 steps. The input and output matches, which were used during the power sweep,

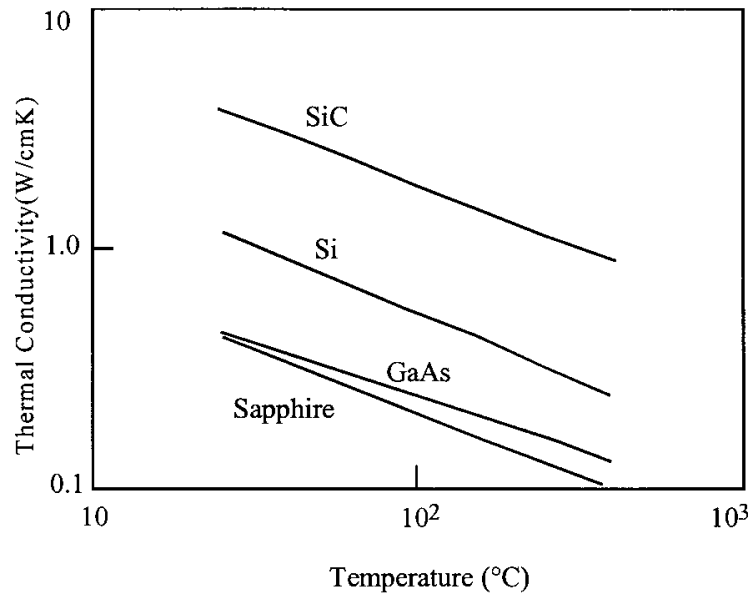

Fig. 13. Thermal conductivity vs. temperature for $\mathrm{SiC}$ and sapphire. After Ref. [46].

were determined by iterating between source and load pulls. The output match was selected to optimize the output power and the input match was selected to maximize the delivered power. The devices were biased to $V_{\mathrm{DS}}=15 \mathrm{~V}$ and $V_{\mathrm{GS}}=-2.5 \mathrm{~V}$. The $I_{\mathrm{DS}}$ at this bias was approximately $20 \mathrm{~mA}$, which corresponds to 260 $\mathrm{mA} \mathrm{mm} \mathrm{m}^{-1}$. Devices exhibited $6 \mathrm{~dB}$ gain for various input levels. The maximum output power was 20.6 $\mathrm{dBm}$ and the peak power added efficiency (PAE) was $17.5 \%$. This corresponds to a normalized output power density of $1.5 \mathrm{~W} \mathrm{~mm}^{-1}$. In general, devices on sapphire substrates suffer from the low thermal conductivity of sapphire substrates and exhibit negative differential resistance in the output characteristics. Remedies include better heat sinking by flip-chip mounting and the use of high resistivity $4 \mathrm{H}-\mathrm{SiC}$ substrates, which provide good thermal conductivity but are hard to obtain.

GaN MODFET devices that have been grown in the author's laboratory on conducting $6 \mathrm{H}-\mathrm{SiC}$ substrates exhibited output characteristics that lacked the negative resistance (they exhibited good heat sinking). There have subsequently been a few reports of MODFET power devices on high resistivity $\mathrm{SiC}$ $[16,19,50]$ and $p$-type SiC [18] substrates with phenomenal improvement in power handling capability notwithstanding the rapid progress on sapphire substrates. On sapphire, recent $0.7 \mu \mathrm{m}$ gate-length $\mathrm{Al}_{0.5} \mathrm{Ga}_{0.5} \mathrm{~N} /$ GaN MODFETs exhibited a current density of $1 \mathrm{~A}$ $\mathrm{mm}^{-1}$, three-terminal breakdown voltages up to $200 \mathrm{~V}$ and $\mathrm{cw}$ power densities of 2.84 and $2.57 \mathrm{~W} \mathrm{~mm}^{-1}$ at 8 and $10 \mathrm{GHz}$, respectively, representing a marked performance improvement for GaN-based FETs.

Unprecedented power levels are being achieved with near-half-micron gate lengths. With $0.7 \mu \mathrm{m}$ gate length devices on $\mathrm{SiC}$ substrates, where the gate-source spa- 


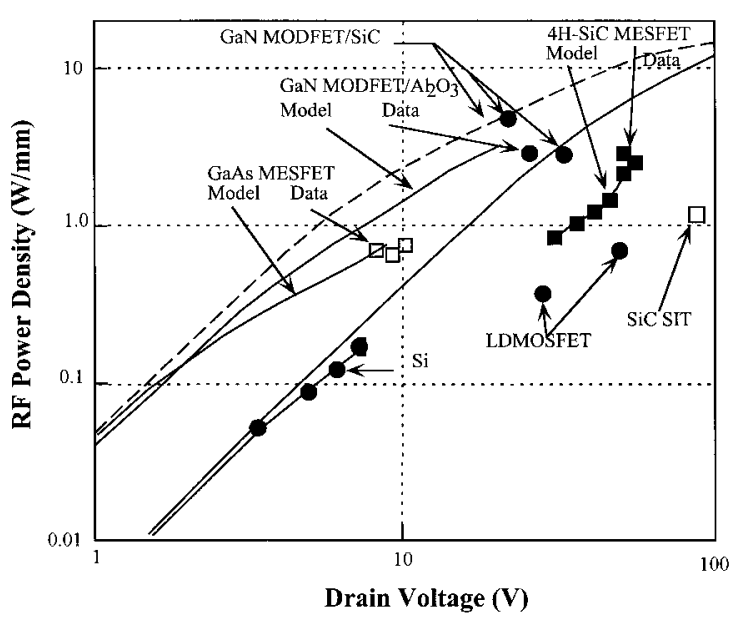

Fig. 14. Simulated and experimental rf power density data for $\mathrm{Si}, \mathrm{GaAs}, \mathrm{SiC}$ and GaN FETs. After Ref. [46].

cing and gate-drain spacing were 0.5 and $0.8 \mu \mathrm{m}$, respectively, a total output power of $2.3 \mathrm{~W}$ in a device with a $1.28 \mathrm{~mm}$ gate periphery has been obtained [19]. The power gain at the $2.3 \mathrm{~W}$ output power point was $3.6 \mathrm{~dB}$ with a PAE of $13.3 \%$. The power measurement was conducted at a drain voltage of $33 \mathrm{~V}$. The current and power gain cutoff frequencies were 15 and 42 $\mathrm{GHz}$. The contact resistance, though not the best, was between 2.6 and $3.5 \Omega \mathrm{mm}$. The maximum normalized transconductance was $270 \mathrm{mS} \mathrm{mm} \mathrm{mm}^{-1}$ and the drain current was $293 \mathrm{~mA} \mathrm{~mm}^{-1}$.

In power devices, the thermal limitation can never be eliminated completely as is the case in nitride devices as well particularly when fabricated on sapphire substrates with a thermal conductivity of only approximately $0.3 \mathrm{~W} \mathrm{~cm} \mathrm{~cm}^{-1} \mathrm{~K}$. Inclusion of thermal limitations leads to results shown in Fig. 14 for devices that compete in the high-power device arena [15]. Since new device developments do in general compete with existing and alternative technologies, a brief account of competing technologies for power arena is given below. The $\mathrm{Si}$ metal semiconductor FET (MESFET) analytical curve, modeled for its simplicity, is slightly above the $\mathrm{SiC}$ analytical curve and indicates a maximum power density of $0.35 \mathrm{~W} \mathrm{~mm}^{-1}$ at $V_{\mathrm{dS}}=7$ $\mathrm{V}$ which is slightly lower than $0.39 \mathrm{~W} \mathrm{~mm}^{-1}$. Since $\mathrm{Si}$ rf MESFETs are unavailable, commercial Si rf metaloxide semiconductor FET (MOSFET) results were used for comparison instead. At low voltages, the $\mathrm{Si}$ MOSFET data parallel the analytical curve suggesting the validity of the functional dependence of power density on drain voltage. Also shown are two higher power density data points $0.4 \mathrm{~W} \mathrm{~mm} \mathrm{~mm}^{-1}, V_{\mathrm{dS}}=28 \mathrm{~V}$ and $0.87 \mathrm{~W} \mathrm{~mm} \mathrm{~mm}^{-1}, V_{\mathrm{dS}}=48 \mathrm{~V}$. These higher power densities were obtained with specially designed rf power MOSFETs that incorporate a lightly doped drain and field plates that significantly increase the breakdown voltage.

The GaAs analytical curve shows the highest power density of all of the devices at the lowest voltages primarily because of the higher electron mobility of GaAs. However, the low breakdown field limits the GaAs MESFET's drain voltage to about $8 \mathrm{~V}$ and

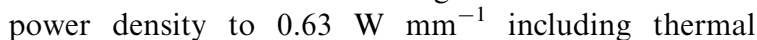
effects. Typical commercially available GaAs MESFET power densities are below $1 \mathrm{~W} \mathrm{~mm}^{-1}$. However, highperformance GaAs FETs with more complex device cross sections have achieved power densities as high as $1.4 \mathrm{~W} \mathrm{~mm}^{-1}$ at $18 \mathrm{~V}$. With even better results in recent pseudomorphic MODFETs at $100 \mathrm{~V}$, the $\mathrm{SiC}$ MESFET has calculated maximum power densities of $7.96 \mathrm{~W} \mathrm{~mm}^{-1}$ with thermal effects and $9.7 \mathrm{~W} \mathrm{~mm}^{-1}$ without thermal effects. The highest demonstrated $\mathrm{cw}$ power density $3.3 \mathrm{~W} \mathrm{~mm} \mathrm{~m}^{-1}\left(V_{\mathrm{ds}}=50 \mathrm{~V}\right)$ for a $\mathrm{SiC}$ MESFET [51] is also shown for comparison. Additional $\mathrm{SiC}$ data again illustrate that the functional dependence of power density on drain voltage. We should caution that while the power density figure can be used during the evolution process, eventually the total power figure must prevail. Normalized power density measurements, though frequently reported (a trap the present author also fell into), are often misleading because smaller gate widths naturally lead to larger power densities [16]. This experimental datum point is actually slightly higher than the simulated result, possibly because of the very small size of the experimental device (100 $\mu \mathrm{m}$ width).

The GaN results of analytical models are highly dependent on the thermal conductivity of the substrate. With a sapphire substrate, the device is severely thermally limited to $2.24 \mathrm{~W} \mathrm{~mm} \mathrm{~m}^{-1}$ at $30 \mathrm{~V}$ with a resulting channel temperature over $400^{\circ} \mathrm{C}$. However, with a $\mathrm{SiC}$ substrate the analysis predicts that a $\mathrm{GaN}$ MODFET could achieve $15.5 \mathrm{~W} \mathrm{~mm}^{-1}$ at $100 \mathrm{~V}$ while keeping the channel temperature at about $300^{\circ} \mathrm{C}$ [46]. To date, the highest power density achieved for a 0.45 $\mu \mathrm{m} \times 125 \mu \mathrm{m} \mathrm{GaN} \mathrm{MODFET} \mathrm{is} 6.8 \mathrm{~W} \mathrm{~mm}^{-1}$ at 10 $\mathrm{GHz}$ with a PAE of $52.4 \%$ and an associated gain of $10.65 \mathrm{~dB}$. At $16 \mathrm{GHz}$, the same figures, in the same order, are $4.4 \mathrm{~W} \mathrm{~mm}^{-1}, 27.3 \%$ and $6.9 \mathrm{~dB}$ [16]. The key to further improvements lies with our ability to control the polarity of the films, to prepare domain free material and to reduce defects. GaN MODFETs technology has spawned so fast that wideband amplifier reports also are beginning to emerge [52].

Efforts to enhance upon the reported MODFET results are relentlessly continuing. MODFETs require high resistivity buffer layers of GaN. Since the background doping levels in $\mathrm{GaN}$ are not extremely low, there are two approaches one can employ toward that goal. One is to compensate $n$-type background by stoichiometry (native levels) [53] and the other is by dop- 
Table 2

Sheet carrier density and mobility values at room temperature and $77 \mathrm{~K}$ for a number of $2 \mathrm{D}$ electron gas samples reported in the literature by molecular beam epitaxy except the indicated sample. Mobility figures obtained informally from at least two other laboratories are consistent with those reported in the table

\begin{tabular}{lllll}
\hline Ref & Al content $(\%)$ & $n\left(10^{12} \mathrm{~cm}^{-2}\right), 300 \mathrm{~K}: 77 \mathrm{~K}$ & $\mu\left(\mathrm{cm}^{2} / \mathrm{V} \mathrm{s}\right), 300 \mathrm{~K}$ & $\mu\left(\mathrm{cm}^{2} / \mathrm{V} \mathrm{s}\right), 77 \mathrm{~K}$ \\
\hline Gaska et al. [58] & 0.20 & $>7$ & 2000 & $4500,9000(?)$ \\
Li et al. [59] & 0.20 & $\sim 100: \sim 10$ & 750 & 4070 \\
Dimitrov et al. [55] & $0.23-0.4$ & $4.5: 4.2$ & 1150 & 3450 \\
Webb et al. [56] & 0.07 & $4.9: \sim 4.9$ & 1211 & 5660 \\
\hline
\end{tabular}

\footnotetext{
${ }^{\mathrm{a}}$ By organometallic vapor phase epitaxy. The ? mark is meant to indicate that the temperature dependence of the mobility appears somewhat extraordinary.
}

ing with impurities that create an acceptor like level. In fact, the very first LED, reported nearly 30 years ago, in GaN was an MIS type where I stood for insulating GaN [54]. Essentially, a nominally $n$-type GaN was doped with $\mathrm{Zn}$ during growth. Acceptor-like centers introduced by $\mathrm{Zn}$ compensated electrons in the system leading to high resistivity. Among other dopants used for compensating $\mathrm{GaN}$ is $\mathrm{Mg}$ in the case of GaN only bufer layers [55]. Very recently, C was used to compensate the electrons in the system in an effort to obtain high resistivity buffer layers [56]. $\mathrm{AlGaN} / \mathrm{GaN}$ heterostructures grown on the C-doped semi-insulating GaN base layers exhibited a high mobility two-dimensional electron gas at the hetero-interface, with room temperature mobilities typically between 1000 and $1200 \mathrm{~cm}^{2} / \mathrm{V} \mathrm{s}$ and liquid nitrogen temperature mobilities up to $5660 \mathrm{~cm}^{2} / \mathrm{V} \mathrm{s}$. The carrier density for the sample exhibiting a sheet carrier density of approximately $5 \times 10^{12} \mathrm{~cm}^{-2}$ was almost constant, with less than $3 \%$ change over the measured temperature range. This is in contrast to generally $3 \times 10^{13}$ $\mathrm{cm}^{-2}$ reported by many groups which in and of itself is very interesting in that polarization charge which accounts for the $3 \times 10^{13} \mathrm{~cm}^{-2}$-like sheet carrier concentrations. Mobilities reported by several groups in GaN/AlGaN modulation doped structures have been tabulated in Table 2. Some of the results reported are obtained by reactive MBE using ammonia as the nitrogen source. Others utilized nitrogen activated by an rf plasma source. One thing is common in that MBE is able to produce increasingly high mobility modulation doped structures with uniformity that bodes well for MODFET devices with high yield. The polarization issues in conjunction with modulation doped structure have been treated well by Ambacher et al. [57].

\subsection{Conjecture}

An attempt to conjecture about what may be in the wing is well warranted. In conventional III-V semiconductors, the room temperature electron mobility in best modulation doped films is limited by polar optic phonon scattering mobility. Experimentally, AlGaAs/ GaAs modulation doped layer mobilities are nearly identical to very high quality GaAs bulk mobilities at room temperature. In $\mathrm{GaN}$ based modulation doped structures, however, the sheet mobility at the interface is nearly a factor of two higher than predicted bulk mobilities. Although, one can invoke many mechanisms as to why, such as less scattering from scattering sources due to two dimensionality, an argument can also be made that perhaps the predictions need to be fine tuned. Regardless of these arguments and counterarguments, experimental work is progressing well and more and more groups are reporting mobilities which are consistently increasing with time. All of these developments bode very well for MODFETs based on $\mathrm{GaN}$ in that yet higher mobilities, higher MODFET transconductances, higher cut-off frequencies and power levels over what have thus far been reported will surely be attained.

\section{Conclusions}

In conclusion, GaN and related materials exhibit optical and transport characteristics that are very well suited for visible opto-electronic devices and electronic devices (relying on electron transport). This is evident by successful LEDs and lasers and the recent high power MODFETs with record power performance which supplanted all the other competing semiconductors at microwave frequencies. Assuming that some day the intrinsic dielectric breakdown strength can be achieved, applicable figures of merit for switching/ power device indicate that nitride semiconductors would be equally successful. Recent theoretical and experimental investigations indicate that polarization induced charge is important in nitride semiconductors with device implications and that spontaneous polarization is dominant in many cases over the strain induced piezoelectric polarization in typical $\mathrm{GaN} /$ AlGaN structures. This dominance would increase if the AlGaN grown on GaN relaxes in full or in part. 
However, the differential spontaneous polarization between $\mathrm{GaN}$ and $\mathrm{InN}$ is much smaller than that of GaN/AlN interfaces. Assuming that the InGaN on $\mathrm{GaN}$ is fully strained, the piezoelectric component of polarization would dominate. Full or partial relaxation of InGaN on $\mathrm{GaN}$ due to defects and inhomogeneities such as compositional fluctuations would reduce this piezoelectric polarization substantially. For now, optical transitions in $\mathrm{GaN} / \mathrm{AlGaN}$ quantum wells investigated support the assertion that spontaneous polarization is dominant in those structures and must not be lumped with piezoelectric polarization. Heterostructures based on GaN have paved the way to $\mathrm{AlGaN} / \mathrm{GaN}$ modulation doped field effect transistors with an X-band cw power level of $4 \mathrm{~W}$ at $10 \mathrm{GHz}$ in a $2 \mathrm{~mm}$ gate periphery device and a $29 \%$ power added efficiency. The current and power gain cutoff frequencies for devices with approximately $0.25 \mu \mathrm{m}$ gate lengths are over 50 and $100 \mathrm{GHz}$, respectively. What is also very exciting is the steady progress made in improving the quality of the modulation doped material. At the time of this writing, several laboratories have reported room temperature mobilities well in excess of $1000 \mathrm{~cm}^{2} \mathrm{~V}^{-1} \mathrm{~s}^{-1}$ at room temperature with $77 \mathrm{~K}$ values being on the heels of $10,000 \mathrm{~cm}^{2} \mathrm{~V}^{-1} \mathrm{~s}^{-1}$. Therefore, the time-honored adage applies here very well in that every day will bring about new and better results yet. Another bright image is also apparent on the horizon for MBE in that very high electron mobilities coupled with a high degree of uniformity bode well for the technique for producing electronic devices on commercial scale as it has done for many of the other compound semiconductor devices.

\section{Acknowledgements}

The work at VCU is supported by the Air Force Office of Scientific Research and Office of Naval Research under the direction of Dr. G.L. Witt, Dr. C.E.C. Wood, Dr. Y.S. Park and Mr. M. Yoder. The authors would like to thank Professor H. Jiang and Professor Y. Lin of Kansas State, and D. Pavlidis of University of Michigan, A. Di Carlo and P. Lugli of University Tor Vergata (Italy) for many enlightening discussions and collaborations. They are particularly indebted to Professor Walter Lambrecht for many clarifying discussions on polarization issues and collaborations. Professor S. Hersee of the University of New Mexico provided the essence of Fig. 2. Finally, the authors are grateful to Professor S. Pearton for his encouragement.

\section{References}

[1] Morkoç H. Nitride semiconductors and devices. Heidelberg: Springer Verlag, 1999.

[2] Mohammad SN, Morkoç H. Progr Quantum Electr 1996;20(5, 6):361-525.

[3] Mohammad SN, Salvador A, Morkoç H. Proc IEEE 1995;83:1306-55.

[4] Morkoç H, Strite S, Gao GB, Lin ME, Sverdlov B, Burns M. J Appl Phys Rev 1994;76(3):1363-98.

[5] Strite ST, Morkoç H. GaN. J Vac Sci Technol B 1992;10:1237-66.

[6] Duffy MT, Wang CC, O'Clock GD, McFarlane III SH, Zanzucchi PJ. J Electr Mat 1973;2:359.

[7] Razeghi M, Rogalski A. J Appl Phys 1996;79:7433-73.

[8] Xu GY, Salvador A, Kim W, Fan Z, Lu C, Tang H, Morkoç H, Smith G, Estes M, Goldenberg B, Yang W, Krishnankutty S. Appl Phys Lett 1997;71(15):2154-6.

[9] Fritz IJ, Drummond TJ. Electr Lett 1995;31:68-9.

[10] Nakamura S, Mukai T, Senoh M. Appl Phys Lett 1994;64:1687-9.

[11] Morkoç H, Mohammad SN. Sci Mag 1995;267:51-5.

[12] Morkoç H, Mohammad SN. Light emitting diodes. In: Webster $\mathbf{J}$, editor. Wiley encyclopedia of electrical engineering and electronics engineering. Wiley, 1999.

[13] Nakamura $S$, Senoh $M$, Nagahama $N$, Iwara $N$, Yamada T, Matsushita T, Kiyoku H, Sugimoto Y, Kozaki T, Umemoto H, Sano M, Chocho K. Jpn J Appl Phys 1997;38:L1578.

[14] Morkoç H. IEEE J Sel Topics Quantum Electr 1998;4(3):537-49.

[15] Morkoç H. Beyond SiC! III-V nitride based heterostructures and devices. In: Park YS, editor. SiC materials and devices. Willardson and Beer series, vol. 52. Academic Press, 1998:307-94.

[16] Sheppard ST, Doverspike K, Pribble WL, Allen ST, Palmour JW, Kehias LT, Jenkins TJ. IEEE Electron Dev Lett 1999;20(4):161.

[17] Wu Y-F, Keller BP, Fini P, Keller S, Jenkins TJ, Kehias LT, Denbaars SP, Mishra UK. IEEE Electron Dev Lett 1998;19(2):50-3.

[18] Ping AT, Chen Q, Yang JW, Khan MA, Adesida I. IEEE Electron Device Lett 1998;19(2):54-6.

[19] Sullivan GJ, Chen MY, Higgins JA, Yang JW, Chen Q, Pierson RL, McDermott BT. IEEE Electron Dev Lett 1998;19:198-9.

[20] Takeuchi T, Wetzel C, Yamaguchi S, Sakai H, Amano H, Akasaki I, Kaneko Y, Nakagawa S, Yamaoka Y, Yamada N. Appl Phys Lett 1998;73:1691.

[21] Im JS, Kollmer H, Off J, Sohmer A, Scholz F, Hangleiter A. Phys Rev B 1998;57:R9435.

[22] Takeuchi T, Wetzel C, Yamaguchi S, Sakai H, Amano H, Akasaki I, Kaneko Y, Nakagawa S, Yamaoka Y, Yamada N. Appl Phys Lett 1998;73:1691.

[23] Kim HS, Lin JY, Jiang HX, Chow WW, Botchkarev AE, Morkoç H. Appl Phys Lett, 1999 [in press].

[24] Leroux M, Grandjean N, Massies J, Gil B, Lefebvre P, Bigenwald P. Phys Rev B, 1999 [in press].

[25] Cingolani R, Botchkarev A, Tang H, Morkoç H, Coliì G, Lomascolo M, Di Carlo A, Lugli P. Phys Rev B, 1999 [in press]. 
[26] Yu ET, Sullivan GJ, Asbeck PM, Wang CD, Qiao D, Lau SS. Appl Phys Lett 1997;71:2794.

[27] Asbeck PM, Yu ET, Lau SS, Sullivan GJ, Van Hove J, Redwing J. Electr Lett 1997;33(14):1230.

[28] King-Smith RD, Vanderbilt D. Phys Rev B 1990;47:1651.

[29] Resta R. Rev Mod Phys 1994;66:899.

[30] Bernardini F, Fiorentini V, Vanderbilt D. Phys Rev B 1997;56:R10024.

[31] Bernardini F, Fiorentini V. Phys Rev B 1998;57(16):1-4.

[32] Kim K, Lambrecht WRL, Segall B. Phys Rev B 1997;56:7018.

[33] Morkoç H, Ünlü H, Ji G. Fundamentals and technology of MODFETs, II. Wiley, 1991.

[34] Martin GA, Strite S, Botchkarev A, Agarwal A, Rockett A, Morkoç H, Lambrecht WRL, Segall B. Appl Phys Lett 1994;65:610.

[35] Martin GA, Botchkarev A, Agarwal A, Rockett A, Morkoç H. Appl Phys Lett 1996;68:2541.

[36] Martin GA. Semiconductor electronic band alignment at heterojunctions of wurtzite AlN, GaN and InN. Ph.D. thesis, Department of Physics, University of Illinois, 1996.

[37] Gil B, Lefebvre P, Allègre J, Mathieu H, Grandjean N, Leroux M, Massies J, Bigenwald P, Christol P. Phys Rev B, 1999 [in press].

[38] Bastard G. Wave mechanics applied to semiconductor heterostructures. Paris: Edition de Physique, 1987.

[39] Singh J. In: Semiconductor optoelectronics, 1995.

[40] Della Sala F, DiCarlo A, Lugli P, Bernardini F, Fiorentini V, Scholz R, Jancu JM. Appl Phys Lett, 1999 [in press].

[41] Jancu JM, Scholz R, Beltram F, Bassani F. Phys Rev B 1995;57:6493.

[42] Morkoç H, Klem J, Masselink WT, Herderson T, Ketterson A. Semiconductor device with strained InGaAs layer. Patent US, \#4,827,320.

[43] Özgür A, Kim W, Fan Z, Mohammad SN, Botchkarev A, Salvador A, Sverdlov B, Morkoç H. Electr Lett 1995;31(16):1389-90.
[44] Drummond TJ, Morkoç H, Lee K, Shur M. IEEE Electr Dev Lett 1982;EDL-3:338-41.

[45] Stengel F, Mohammad SN, Morkoç H. J Appl Phys 1996;80(5):3031-42.

[46] Weitzel C, Pond L, Moore K, Bhatnagar M. Effect of device temperature on $\mathrm{rf}$ FET power density. In: Proc. of silicon carbide, III-nitrides and related materials, ICSI, August 1997, Stockholm, Sweden. Materials science forum, vols. 264-268. Trans Publications, 1998:907-12.

[47] Moloney M, Ponse F, Morkoç H. IEEE Trans Electron Dev 1985;ED-32(9):1675-84.

[48] Smith L. Solid-St Commun 1986;57:919.

[49] Bykhovski, Gelmont B, Shur M. J Appl Phys 1993; 74:6734.

[50] Binari S, Redwing JM, Kelner G, Kruppa W. Electron Lett 1997;33(3):242-3.

[51] Moore KE, Weitzel CE, Nordquist KJ, Pond III LL, Palmour JW, Allen S, Carter Jr. CH. IEEE Electron Dev Lett 1997;18:69-70.

[52] Xu JJ, Wu Y-F, Keller S, Parish G, Thibeault BJ, Hekman S, Mishra UK, York RA. IEEE Microwave Guided Wave Lett, 1999 [in press].

[53] Look DC, Reynolds DC, Kim W, Aktas Ö, Botchkarev A, Salvador A, Morkoç H. J Appl Phys 1996;80(5):29602.

[54] Pankove JI, Miller EI, Berkeyheiser JE. RCA Rev 1971;32:383.

[55] Dimitrov R, Mitchell A, Wittmer L, Ambacher O, Stutzmann M. Appl Phys Lett, 1999 [in press].

[56] Webb JB, Tang H, Rolfe S, Bardwell J. Appl Phys Lett, 1999 [submitted].

[57] Ambacher O, Smart J, Shealy JR, Weimann NG, Chu K, Murphy M, Schaff WJ, Eastman LF. J Appl Phys 1999;85 [in press].

[58] Gaska R, Shur MS, Bykhovski D, Orlov AO, Snider GL. Appl Phys Lett 1999;74(2):287.

[59] Li LK, Alperin J, Wang WI, Look DC, Reynolds DC. J Vac Sci Technol B 1998;16:1275. 